\title{
The Multi-Isotope Process (MIP) Monitor Project: FY13 Final Report
}

\author{
DE Meier \\ K Unlu \\ JB Coble \\ S Landsberger \\ LW McDonald \\ SE Bender \\ DV Jordan \\ KJ Dayman \\ JB Forrester \\ DD Reilly \\ JM Schwantes
}

September 2013

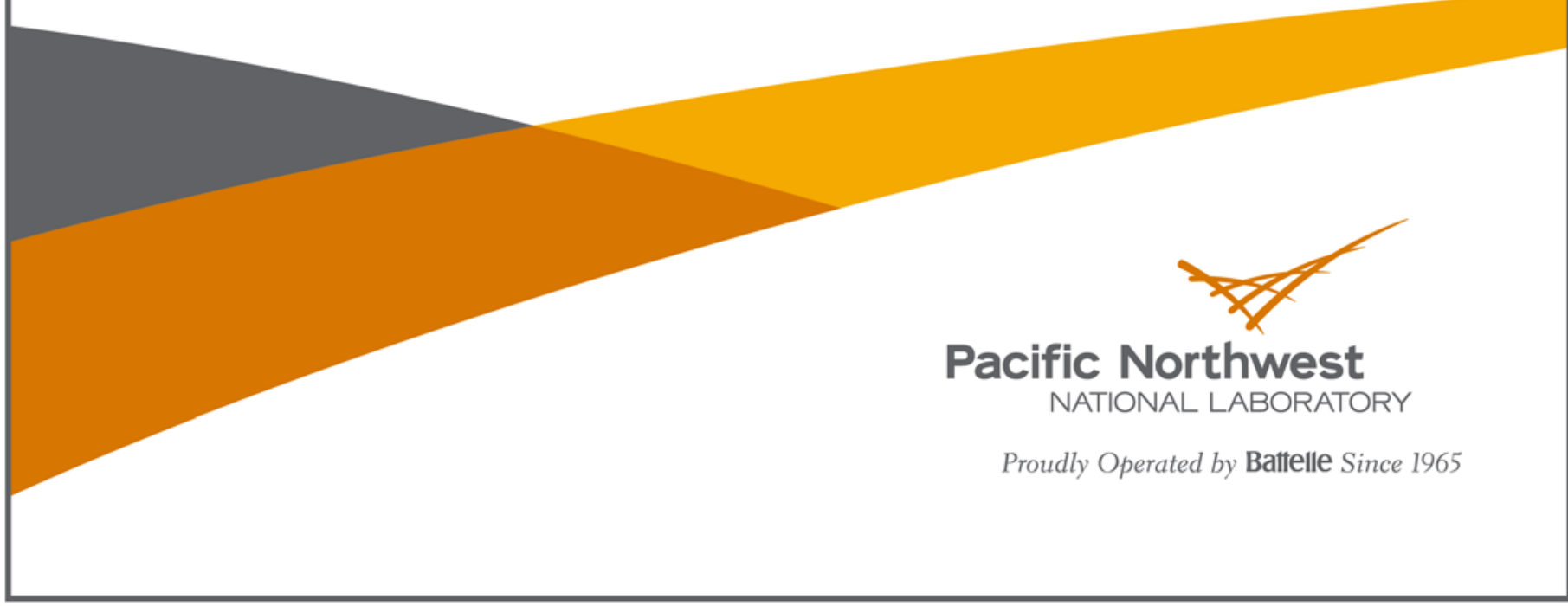




\title{
DISCLAIMER
}

This report was prepared as an account of work sponsored by an agency of the United States Government. Neither the United States Government nor any agency thereof, nor Battelle Memorial Institute, nor any of their employees, makes any warranty, express or implied, or assumes any legal liability or responsibility for the accuracy, completeness, or usefulness of any information, apparatus, product, or process disclosed, or represents that its use would not infringe privately owned rights. Reference herein to any specific commercial product, process, or service by trade name, trademark, manufacturer, or otherwise does not necessarily constitute or imply its endorsement, recommendation, or favoring by the United States Government or any agency thereof, or Battelle Memorial Institute. The views and opinions of authors expressed herein do not necessarily state or reflect those of the United States Government or any agency thereof.

\author{
PACIFIC NORTHWEST NATIONAL LABORATORY \\ operated by \\ BATTELLE \\ for the \\ UNITED STATES DEPARTMENT OF ENERGY \\ under Contract DE-AC05-76RL01830
}

Printed in the United States of America
Available to DOE and DOE contractors from the Office of Scientific and Technical Information,
P.O. Box 62, Oak Ridge, TN 37831-0062;
ph: (865) 576-8401
fax: $(865)$ 576-5728
email: reports@adonis.osti.gov

\begin{abstract}
Available to the public from the National Technical Information Service, U.S. Department of Commerce, 5285 Port Royal Rd., Springfield, VA 22161 ph: (800) 553-6847 fax: $(703) 605-6900$ email: orders@ntis.fedworld.gov online ordering: http://www.ntis.gov/ordering.htm
\end{abstract}

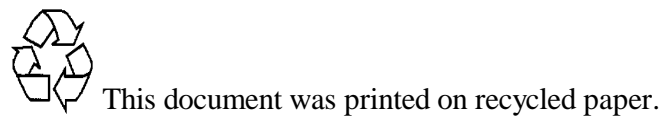




\section{The Multi-Isotope Process (MIP) Monitor Project: FY13 Final Report}

September 2013

Prepared for

the U.S. Department of Energy

under Contract DE-AC05-76RL01830

Pacific Northwest National Laboratory

Richland, Washington 99352 



\section{Summary}

The Multi-Isotope Process (MIP) Monitor provides an efficient approach to monitoring the process conditions in reprocessing facilities in support of the goal of "... (minimization of) the risks of nuclear proliferation and terrorism." The MIP Monitor measures the distribution of the radioactive isotopes in product and waste streams of a nuclear reprocessing facility. These isotopes are monitored online by gamma spectrometry and compared, in near-real-time, to spectral patterns representing "normal" process conditions using multivariate analysis and pattern recognition algorithms. The combination of multivariate analysis and gamma spectroscopy allows us to detect small changes in the gamma spectrum, which may indicate changes in process conditions. By targeting multiple gamma-emitting indicator isotopes, the MIP Monitor approach is compatible with the use of small, portable, relatively high-resolution gamma detectors that may be easily deployed throughout an existing facility. The automated multivariate analysis can provide a level of data obscurity, giving a built-in information barrier to protect sensitive or proprietary operational data. Proof-of-concept simulations and experiments have been performed in previous years to demonstrate the validity of this tool in a laboratory setting for systems representing aqueous reprocessing facilities. However, pyroprocessing is emerging as an alternative to aqueous reprocessing techniques. This report describes research to evaluate the applicability of the MIP Monitor approach to pyroprocessing systems during fiscal year 2013 (FY13). Specific aspects of research completed in FY13 include:

- The applicability and efficacy of the MIP Monitor approach to key locations in pyroprocessing facilities were investigated through models and simulations.

- The bench-scale flow loop was modified to provide better process control and to use event mode counting in late FY12. Data were collected using this system to simulation mixing of dilute spent fuel samples. These data were analyzed to evaluate the ability to differentiate three fuel types during mixing.

- The framework to characterize uncertainty propagation in the multivariate analysis of gamma spectra developed in FY12 was further evaluated using collected gamma spectra. Previous results from spectra collected with an HPGe detector were compared to those from spectra collected with $\mathrm{LaBr}_{3}$.

- Preliminary plans were developed that would establish R\&D process monitoring at the H-Canyon site at SRS. Modeling of the detection systems and multivariate analysis of the acquired data were performed.

- Efforts continue to engage domestic and international partners with the intent to collaborate in order to gain access to large sets of reprocessing data for further MIP Monitor development.

- The MIP Monitor research and development was presented and published in several forums, including several related to the MIP Monitor that were funded either internal to the lab or whose funding was shared between U.S. Department of Energy (DOE) programs. Lists of key publications and presentations follow. 


\section{Publications}

Coble JB, CR Orton, and JM Schwantes. 2013. "Spent Fuel Characterization with the Multi-Isotope Process Monitor." In Proceedings of the 2013 American Nuclear Society Annual Meeting.

Schwantes JM, SA Bryan, CR Orton, TG Levitskaia, SH Pratt, CG Fraga, and JB Coble. 2013. "Advanced process monitoring safeguards technologies at Pacific Northwest National Laboratory." In Proceedings of ATALANTE 2012 International Conference on Nuclear Chemistry for Sustainable Fuel Cycles.

\section{Presentations}

Presenters indicated in bold.

Coble JB, JM Schwantes, CR Orton, and DV Jordan. 2013. "Online Process Verification with the Multi-Isotope Process Monitor." Presented by Jon Schwantes at 245th American Chemical Society (ACS) National Meeting \& Exposition, New Orleans, LA on April 8, 2013.

Coble JB, CR Orton, and JM Schwantes. 2013. "Used Fuel Characterization with the Multi-Isotope Process Monitor." Presented by Jamie B. Coble at American Nuclear Society Annual Meeting, Atlanta, GA on June 18, 2013.

Schwantes JM, SA Bryan, CR Orton, TG Levitskaia, SH Pratt, CG Fraga, and JB Coble. 2013. "Advanced process monitoring safeguards technologies at Pacific Northwest National Laboratory." In ATALANTE 2012 INTERNATIONAL CONFERENCE ON NUCLEAR CHEMISTRY FOR SUSTAINABLE FUEL CYCLES.

Dayman KJ, CR Orton, JB Coble, and JM Schwantes. 2012. "Characterization of Spent Nuclear Fuel using Multivariate Analysis." Presented by Kenneth Dayman at 2012 ANS Winter Meeting and Nuclear Technology Expo, San Diego, CA on November 12, 2012. 


\section{Contents}

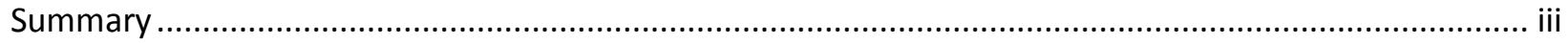

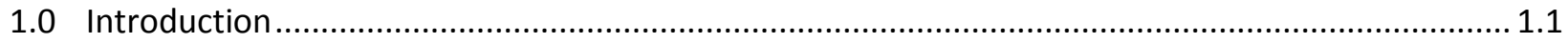

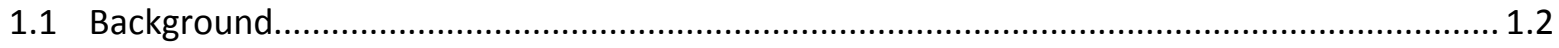

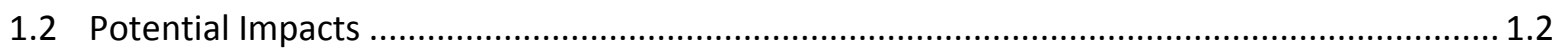

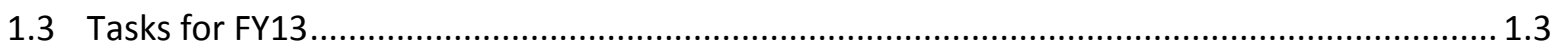

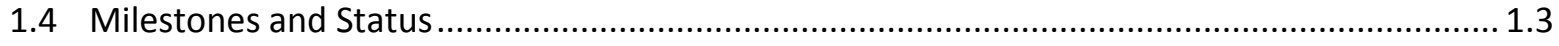

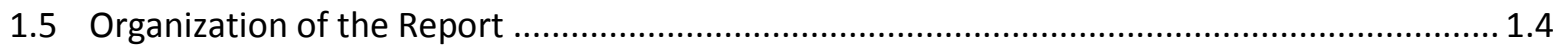

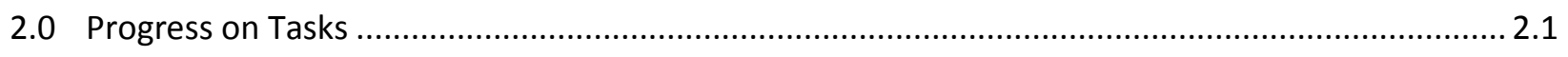

2.1 Task 1: Experimental Efforts to Advance the MIP Monitor Approach ................................... 2.1

2.2 Task 2: Evaluating the Efficacy and Applicability of the MIP Monitor to Pyroprocessing........ 2.7

2.2.1 Anomaly Detection in Pyroprocessing ............................................................2.8

2.2.2 Simulation of Pyroprocessing Process Monitoring with a Medium-resolution

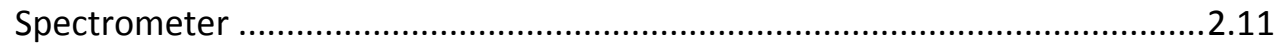

2.3 Task 3: Enhanced Gamma-Ray Spectra Collection Techniques........................................... 2.18

2.4 Task 4: Training of the Next Generation of Nuclear Scientists............................................. 2.18

2.5 Task 5: Coordinating the MIP Monitor Project with Related Activities in Other

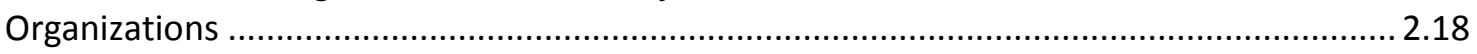

2.6 Task 6: Bench-Scale Flow Loop Experimentation and Analysis ......................................... 2.19

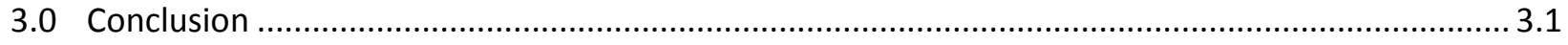

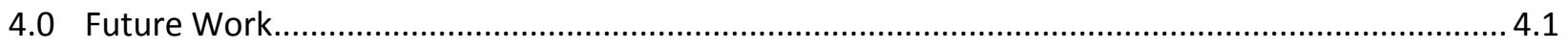

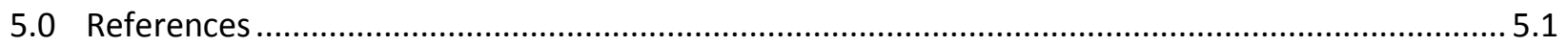




\section{Figures}

Figure 2-1. Comparison of gamma-ray spectra measured using a high-resolution HPGe spectrometer (black) and a medium-resolution $\mathrm{LaBr}_{3}$ scintillation spectrometer (red). The source is one replicate of the sample labeled by the index " 1 " in Table 1. The standoff distance from source to spectrometer is approximately $4 \mathrm{~cm}$, and the dwell times are one hour for the $\mathrm{LaBr}_{3}$, two hours for the HPGe.

Figure 2-3. Statistical Monte Carlo modeling results for application of two multivariate analysis techniques to a set of simulated gamma-ray spectra. The reconstruction precision refers to the RMS fractional deviation of reconstructed from actual source-component activities for a set of 13 synthetic sources consisting of various mixtures of ${ }^{241} \mathrm{Am},{ }^{57} \mathrm{Co}$, and ${ }^{152} \mathrm{Eu}$. The curves join discrete points at integrated spectrum counts of $\{100,200,500, \ldots, 10,000$, $20,000,50,000\}$ and are intended to guide the eye. The results of power-law fits to the sets of \{counts, reconstruction precision\} results are displayed on the plot. Note that an exponent of -0.5 in a power-law fit corresponds to $1 /$ Vcounts variation, as familiar from Poisson counting statistics.

Figure 2-4. Modeled dependence of reconstructed sample BU bias on the nonlinearity in the underlying law mapping BU to spectrum component intensity.

Figure 2-5. Photograph of the Updated Bench-Scale Flow Loop System

Figure 2-6. Clustering of three fuel classes in PC-space.

Figure 2-7. Dynamic data classification results Fuel mixing from ATM 105p to ATM 109 can be accurately classified throughout mixing (top). Fuel mixing from ATM 105a to ATM 109 may be incorrectly classified as ATM 105p (bottom).

\section{Tables}

Table 2-1. Representative radioisotope component activities for sources fabricated at PNNL in FY-2011 and subjected to measurements with HPGe (FY-2011) and $\operatorname{LaBr}_{3}$ (FY-2013) spectrometers for multivariate analysis.

Table 2-2. RMS fractional difference of reconstructed from actual radioisotope component activities for the set of 24 sources ( 8 source types, 3 replicates each) fabricated at PNNL. Separate PLS models were constructed for each of the three radioisotope components. Three latent variables (LV's) were retained in the PLS models. 


\subsection{Introduction}

In April 2010, the U.S. Department of Energy (DOE) issued a report to congress entitled, Nuclear Energy Research and Development Roadmap (DOE 2010). One of the four objectives listed for the DOE's nuclear energy research and development activities is Objective 4: "Understand and minimize the risks of nuclear proliferation and terrorism." The primary focus of this project is to aid in the achievement of this objective.

Conventional nuclear material control and accounting (MC\&A) at bulk handling facilities relies, in large part, on destructive analyses to quantify nuclear material and verify the location of all special nuclear material. Although their accuracy is superb, destructive analyses are extremely resourceintensive, have limited sampling rates, and are associated with a significant time lag from sampling to final reporting. In addition, the error associated with these analyses scales with the size of the facility (OTA 1995, Appendix A). As such, highly precise destructive measurements of special nuclear material alone at large facilities may not be adequate to ensure diversions have not occurred. While it is not likely that more precise destructive measurement techniques will be developed in the near future, intelligent integration of a variety of on-line process monitoring tools capable of near real-time, nondestructive measurements may be successful in adequately safeguarding even the largest envisioned facility. A combination of these techniques utilizing both material accountancy and augmented material control via continuous process flow sheet verification could provide a more robust framework for MC\&A.

Pacific Northwest National Laboratory (PNNL) is currently developing and demonstrating a technology capable of monitoring conditions at a nuclear reprocessing plant on-line, non-destructively, and in near real-time called the Multi-Isotope Process (MIP) Monitor (Smith et al. 2007; Orton et al. 2008; Schwantes et al. 2008; Orton et al. 2009a; Orton et al. 2009b; Schwantes et al. 2009). The MIP Monitor is designed to track changes in the distribution of gamma-emitting elements as evidence that process conditions are changing (Benedict et al. 1981). Online process surveillance by the MIP Monitor is accomplished by coupling the gamma spectra recorded from process streams with multivariate analysis. Multivariate analysis can evaluate the spectral pattern of the gamma emitting nuclides in nearreal-time for statistically relevant signs of significant changes to the process. By watching the process for small unexpected changes, the MIP Monitor can warn of possible process migration to an unintended or undeclared operation. Because the pattern comparison is automatic and autonomous, proprietary operational or fuel information can be protected while assuring process integrity. However, if desired, process conditions can also be quantified using alternate multivariate calibration techniques, making the monitor a potentially valuable part of a comprehensive process control system. In addition, because the monitor is trained on the gamma rays inherent to nuclear fuel, the MIP monitor technique is not inherently limited to aqueous reprocessing. The primary focus of research activities during FY13 was to evaluate the applicability and efficacy of the MIP Monitor approach for pyroprocessing facilities. 


\subsection{Background}

This research began in 2006 with initial scoping studies into the feasibility of the MIP Monitor concept through limited computer simulations. In fiscal year 2008 (FY08), the project was funded and proof-of-concept simulation studies were performed. The results successfully demonstrated the merit of the MIP Monitor approach. In late FY08 and into FY09, with help from the Office of Nonproliferation and International Security (NA-24), proof-of-concept experiments were initiated to confirm model results from the previous years. Since that time, experiments and simulations have been conducted to further characterize and enhance the performance of the MIP Monitoring approach. Experiments to date have focused on providing replicate data (which are required for multivariate analysis), as well as near-real-time data from well-characterized spent fuels. While great strides have been made in understanding how the MIP Monitor reacts to process changes and fuel characteristics, much remains to be done to further characterize the potential of the approach in actual deployments within continuous flow systems.

The overall goals of this project are to continue an assessment of the strengths and limitations of the MIP Monitoring approach for monitoring streams within a nuclear reprocessing facility and to seek opportunities to apply this technology at the pilot and industrial scale. As limitations and strengths are discovered, they will be addressed or capitalized upon in order to develop the MIP Monitor into an effective tool for safeguarding nuclear material and operator process control. The MIP Monitor will then aid in the accomplishment of Objective 4 of the Nuclear Energy Research and Development Roadmap to, “... minimize the risks of nuclear proliferation and terrorism” (DOE 2010).

\subsection{Potential Impacts}

Although the MIP Monitor requires additional research and development prior to deployment at an operating facility, the approach shows potential for providing cost saving improvements to domestic and international MC\&A as well as process monitoring. The MIP Monitor has the potential to increase process monitoring efficiency and efficacy by providing continuous verification of process integrity, which should allow the number and frequency of costly destructive analysis measurements to be reduced at bulk handling facilities. In addition to MC\&A improvements, a mature MIP Monitor may also provide operators with a cost-effective process monitoring control tool. Current monitoring methods require highly radioactive grab samples to be collected from the process stream in order to confirm process conditions. The MIP Monitor could potentially monitor these process variables nondestructively and in near-real-time. Such an approach could significantly reduce operator costs as well as the doses received by analysts.

Research performed in FY12 suggests that the MIP Monitor approach may also be an effective approach to spent fuel burn-up measurements, which are traditionally performed using conventional gamma spectroscopy. By focusing on multivariate patterns rather than the ratios of a few isotopes, changes in spectral patterns might be used to improve on the accuracy of conventional burn-up analysis. Increasing the accuracy of measured burn-up levels of spent fuel will increase the fidelity of the initial plutonium concentration value in spent fuel, providing a more accurate starting point for MC\&A. 


\subsection{Tasks for FY13}

Tasks supporting the accomplishment of the overall goal of the MIP Monitor during FY13 include:

1. Continuation of experimental efforts to more fully test and evaluate the MIP Monitoring approach, taking advantage of the stock of a variety of well characterized radionuclides currently available at the hot cell facility at PNNL using multiple detector types (such as HPGe, CZT, $\mathrm{LaBr}_{3}$, and $\mathrm{Nal}$ ) in different collection modes or geometries.

2. Enhance multivariate techniques for recognizing and quantifying gamma ray spectral patterns and transitioning these efforts to Near-Real-Time applications. Previous research focused on analysis of solvent methods, such as PUREX; however, pyroprocessing is emerging as a strong competitor to solvent-based methods. Research during FY13 focused on evaluating pyroprocessing and assessing how the MIP Monitor can be applied to monitor this process.

3. Working with researchers at Pennsylvania State University to investigate the feasibility of coincidence and anti-coincidence (Compton suppression) measurements as part of the MIP Monitoring approach to both enhance the signal to noise ratio and investigate the as of yet untapped gamma-gamma coincidence signals residing in spent fuel.

4. Training of the next generation of nuclear scientists within the U.S., including coordinating the graduate student efforts at the collaborating universities; effort will be coordinated and in conjunction with that of the NTNFC funding received for FY13. This includes mentoring and support for graduate students at UT-Austin and PSU.

5. Coordinating MPACT MIP Monitor scope with related activities in other programs (e.g. NE's separations campaign, NA-241 safeguards technology development, and PNNL internal investments for developing new process monitoring technologies).

6. Using and modifying a simplified bench-scale flow loop for use evaluating the MIP Monitor performance in a real-time environment using event-mode counting.

\subsection{Milestones and Status}

Two milestones are associated with FY13:

i. Technical memo describing progress (due March 8, 2013; submitted March 7, 2013)

ii. FY13 MIP Monitor Report (due September 27, 2013)

With the submission of this report (milestone 2), all of the milestones for FY13 have been completed. 


\subsection{Organization of the Report}

This report summarizes the research in FY13 to further development of the MIP Monitor. Six tasks were defined for this effort; the following section describes the progress made on each of these tasks. Section 3 summarizes the major accomplishments in FY13. Finally, the work proposed to continue development of the MIP Monitor in FY14 and beyond is summarized in Section 4. 


\subsection{Progress on Tasks}

Six main tasks were defined to continue development of the MIP Monitor. The progress and accomplishments made during FY13 on each of these tasks is summarized in the following sections.

\subsection{Task 1: Experimental Efforts to Advance the MIP Monitor Approach}

The gamma-ray spectrometry assessment task of FY-2013 work in the MIP monitor project represents a follow-on to FY-2012 studies that quantified the impact of Poisson counting statistics on the application of multivariate methods to analysis of gamma-ray spectra. The overall motivation for the FY13 work is to assess the gamma-ray spectrometer requirements for statistically robust, near real-time process monitoring. In general, this performance will depend upon the process stream conditions (material composition and density, radioisotope inventories per unit volume in the process material, presence of attenuating materials and structures around the process material), the measurement geometry (detector size and standoff from the process stream), and the detector response properties (intrinsic efficiency and energy resolution). Systematic and random variations in detector response will tend to add corresponding variations to the measured gamma-ray spectra, variations which in turn can distort or "blur" the results of multivariate analysis applied to the measured spectra in a process-monitoring context. At a minimum, a notional concept of operations (CONOPS) for a MIP monitor should include a specification of the detector type and measurement conditions, an estimate of the dwell times required to achieve a specified confidence in deviation from "normal" process conditions (i.e. anomaly detection), and a budget of the error sources contributing to the uncertainty in the decision chain leading to identification of off-normal process conditions. The MIP project has begun addressing these CONOPS issues by a combination of empirical and computational analyses focused on bounding the potential performance of the MIP under realistic process-stream conditions. Methods applied to the gamma-ray spectrometry assessment in FY-2013 include the following:

1. A comparison of multivariate analyses of measured radioisotope-source spectra accumulated under well-defined laboratory conditions with two different spectrometer types, high-purity germanium (HPGe), a high-resolution (0.25\% energy resolution at $662 \mathrm{keV}$ ) semiconductor material, and lanthanum bromide $\left(\mathrm{LaBr}_{3}\right)$, a scintillator offering medium resolution ( $3 \%$ at 662 $\mathrm{keV})$;

2. Continued assessment of counting-statistics effects, generalizing FY-2012's results using principal component regression (PCR) to a second important class of multivariate technique, partial least squares (PLS), and addressing nonlinear process-variable models;

3. Application of radiation transport and detector-response modeling to compute the response of a candidate MIP gamma-ray spectrometer under a variety of process-stream conditions relevant to pyroprocessing (see Section 2.2).

\section{Comparison of Multivariate Analysis in High-resolution and Medium-resolution Gamma-ray}

\section{Spectrometers}

Previous work (Orton et al. 2011)measured gamma-ray spectra from sets of radioisotope sources fabricated at PNNL. The spectra were collected with a $100 \%$ relative-efficiency HPGe spectrometer, and the spectroscopic data subjected to multivariate analysis. The results were quantified in terms of the ability of a PLS model of several training, or calibration, samples to reconstruct the activities of training samples themselves and to predict the activities of several independent test samples. In FY-2013, these measurements were reprised with a mediumresolution $\mathrm{LaBr}_{3}$ spectrometer. The primary objective of the measurement campaign was to 
compare the multivariate analysis performance for two widely differing detector energy resolutions. Figure 2-1 compares spectra from the two spectrometers for the same source. The training source set consisted of eight samples combining three radioisotopes, ${ }^{241} \mathrm{Am},{ }^{57} \mathrm{Co}$, and

${ }^{152} \mathrm{Eu}$, in varying proportions. The nominal activities of each radioisotope component ranged from 100 to $200 \mu \mathrm{Ci}$ at the time of fabrication in 2011; see Table 2-1. Three physically-distinct replicates of each sample were available for measurement. The raw $\mathrm{LaBr} 3$ spectra were energy-calibrated using independent measurements with gamma-ray "check" sources, emitting gamma-ray lines spanning the energy range of interest for the analysis. We applied PLS analysis to model the variation of the measured spectral content with the underlying "process variables," which in this case were simply the activities of the three radioisotope components used in fabricating a given sample. The PLS model trained on the calibration set yields a reconstructed, or "fitted," value of the sample activities. (Three separate PLS models, constructed independently for the three isotopes, were used in this reconstruction).

compares the multivariate reconstruction accuracy for the $\mathrm{LaBr}_{3}$ spectrometer and the FY11 HPGe spectrometer measurement campaigns. The conclusions of the study to date is that the multivariate performance is comparable for the two spectrometer types, suggesting that the excellent energy resolution of the relatively expensive and operationally resource-intensive (i.e., cryogenic cooling required) HPGe detector type are not essential for successful application of the multivariate analysis approach. A medium-resolution detector material (e.g. $\mathrm{LaBr}_{3}$ or $\mathrm{Nal}$ ) may work reasonably well. It should be noted that gain drifts (i.e. systematic, temperature-induced shifts in the mapping from deposited energy to output spectrum channel number) can affect the energy calibration of scintillation spectrometers, such as $\mathrm{LaBr}_{3}$ and Nal. Mitigating the effects of gain drift would require implementing automatic calibration techniques that could be applied either periodically or continuously (by monitoring, for example, the position of a calibration peak from a built-in radioisotope source). We have not yet attempted to address gain drift effects or mitigation techniques in FY2013 work. One relatively simple possibility for assessing the impact of gain drifts is to simply repeat the PLS analysis for the raw, or uncalibrated, $\mathrm{LaBr}_{3}$ spectra. These spectra exhibit day-to-day variations in gain of order a few percent in the linear channel-to-energy calibration factor (an effect which is effectively removed in the energy calibration procedure).

\begin{tabular}{|l|l|l|l|}
\hline Sample index & \multicolumn{3}{|c|}{ Nominal Activity $(\mu \mathrm{Ci})$} \\
\hline 1 & \multicolumn{1}{|c|}{${ }^{241} \mathrm{Am}$} & ${ }^{57} \mathrm{Co}$ & ${ }^{152} \mathrm{Eu}$ \\
\hline 2 & 200 & 200 & 200 \\
\hline 3 & 200 & 200 & 100 \\
\hline$\ldots$ & 100 & 200 & 100 \\
\hline 8 & & & \\
\hline
\end{tabular}

Table 2-1. Representative radioisotope component activities for sources fabricated at PNNL in FY-2011 and subjected to measurements with HPGe (FY-2011) and $\mathrm{LaBr}_{3}$ (FY-2013) spectrometers for multivariate analysis. 


\begin{tabular}{|l|c|c|c|}
\hline Detector & \multicolumn{3}{|c|}{ PLS Reconstruction Accuracy (\%) } \\
\hline & ${ }^{241} \mathrm{Am}$ & ${ }^{57} \mathrm{Co}$ & ${ }^{152} \mathrm{Eu}$ \\
\hline $\mathrm{HPGe}$ & 5 & 3 & 2 \\
\hline $\mathrm{LaBr}_{3}$ & 4 & 4 & 3 \\
\hline
\end{tabular}

Table 2-2. RMS fractional difference of reconstructed from actual radioisotope component activities for the set of 24 sources (8 source types, 3 replicates each) fabricated at PNNL. Separate PLS models were constructed for each of the three radioisotope components. Three latent variables (LV's) were retained in the PLS models.

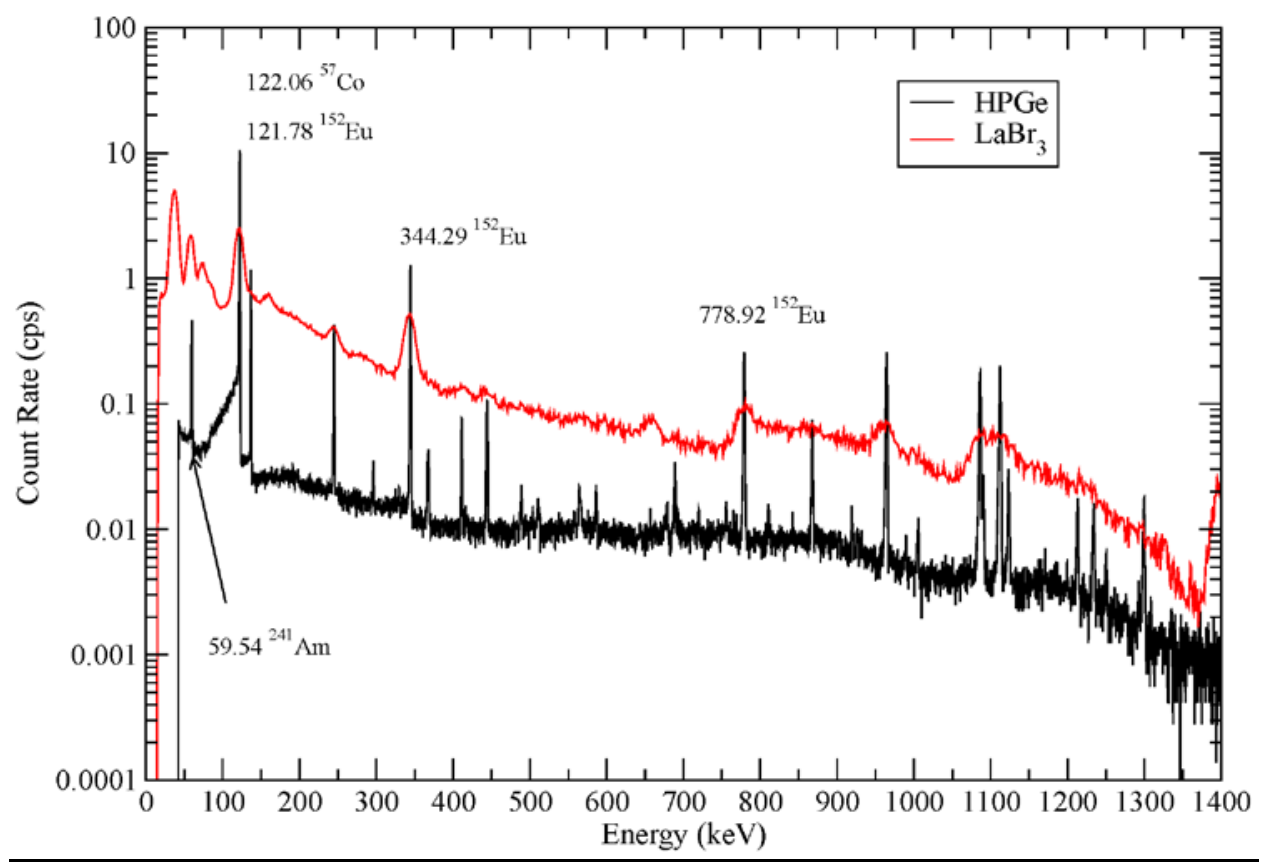

Figure 2-1. Comparison of gamma-ray spectra measured using a high-resolution HPGe spectrometer (black) and a medium-resolution $\mathrm{LaBr}_{3}$ scintillation spectrometer (red). The source is one replicate of the sample labeled by the index " 1 " in Table 2-1. The standoff distance from source to spectrometer is approximately $4 \mathrm{~cm}$, and the dwell times are one hour for the $\mathrm{LaBr}_{3}$, two hours for the HPGe.

\section{Impact of Counting Statistics on PLS-based Multivariate Methods}

Statistical modeling work carried out in FY-2012 addressed the effect of gamma-ray spectrum counting statistics on principal component regression (PCR) techniques. The modeling methodology used synthetic spectra in a notional HPGe spectrometer as the basis for constructing, via Monte Carlo techniques, a relatively large (order 100 to 1000 spectra) ensemble of simulated spectrum sets. Each channel in a given spectrum was sampled from a Poisson statistical distribution, with mean 
corresponding to the number of counts in the synthetic template spectrum. These spectrum sets were then subjected to multivariate analysis, and the reconstruction precision for a set of "test" sample spectra histogrammed. The resulting probability distribution in the reconstructed precision afforded a mapping of the impact on counting "noise" on the multivariate performance, as the mean total counts in the template spectra varies. The specific multivariate method applied to the FY-2012 work was PCR, which amounts to a combination of principal component analysis (PCA) and a suitable linear regression model of the underlying process variables constructed in terms of the PCA scores obtained from analysis of each spectrum. This sufficed for a first pass, but the MIP monitor algorithm developed in FY-2012 for spent fuel classification and quantitative process-variable reconstruction (Coble et al. 2012) actually applies a variant of PLS. Thus in FY-2013, we reprised the statistical modeling studies from FY-2012, this time using PLS, rather than PCR, as the multivariate analysis method.

Figure 2-2 displays the results of the modeling, which displays the reconstruction precision for a set of 13 synthetic samples (each representing a linear combination of emissions from ${ }^{241} \mathrm{Am},{ }^{57} \mathrm{Co}$, and ${ }^{152} \mathrm{Eu}$ ). The solid curves in the plot join points that each represent averages over several thousand Monte Carlo trials. The results indicate that PLS and PCR both exhibit (roughly) a $1 / V$ counts variation in the overall reconstruction precision, suggestive of the variation in single-peak intensity measurement precision with counts familiar from conventional gamma-ray spectrometry (Knoll 2000). In this particular modeling case, the PLS reconstruction ( 3 latent variables per isotope, for a total of 9 parameters retained in the model) out-performs the PCR reconstruction (4 fit terms per isotope) in describing the synthetic data, but the primary point of the investigation is to verify that the variation of reconstructed accuracy with gamma-ray spectrum counts can be roughly approximated by a Poisson single-peak statistical "rule of thumb." This in turn furnishes a quantitative basis for assessing the requisite counting statistics for confident application of more general multivariate algorithms (e.g. the spent fuel algorithm of [Coble 2012]). In practice, we will model the gamma-ray spectrometry response of a notional detector type in a notional MIP monitor application, model the conclusions of MCA analysis for a single exposure time, and then use a suitable scaling law to analytically predict the performance at an arbitrary dwell time, without having to repeat the Monte Carlo statistical modeling each time. 


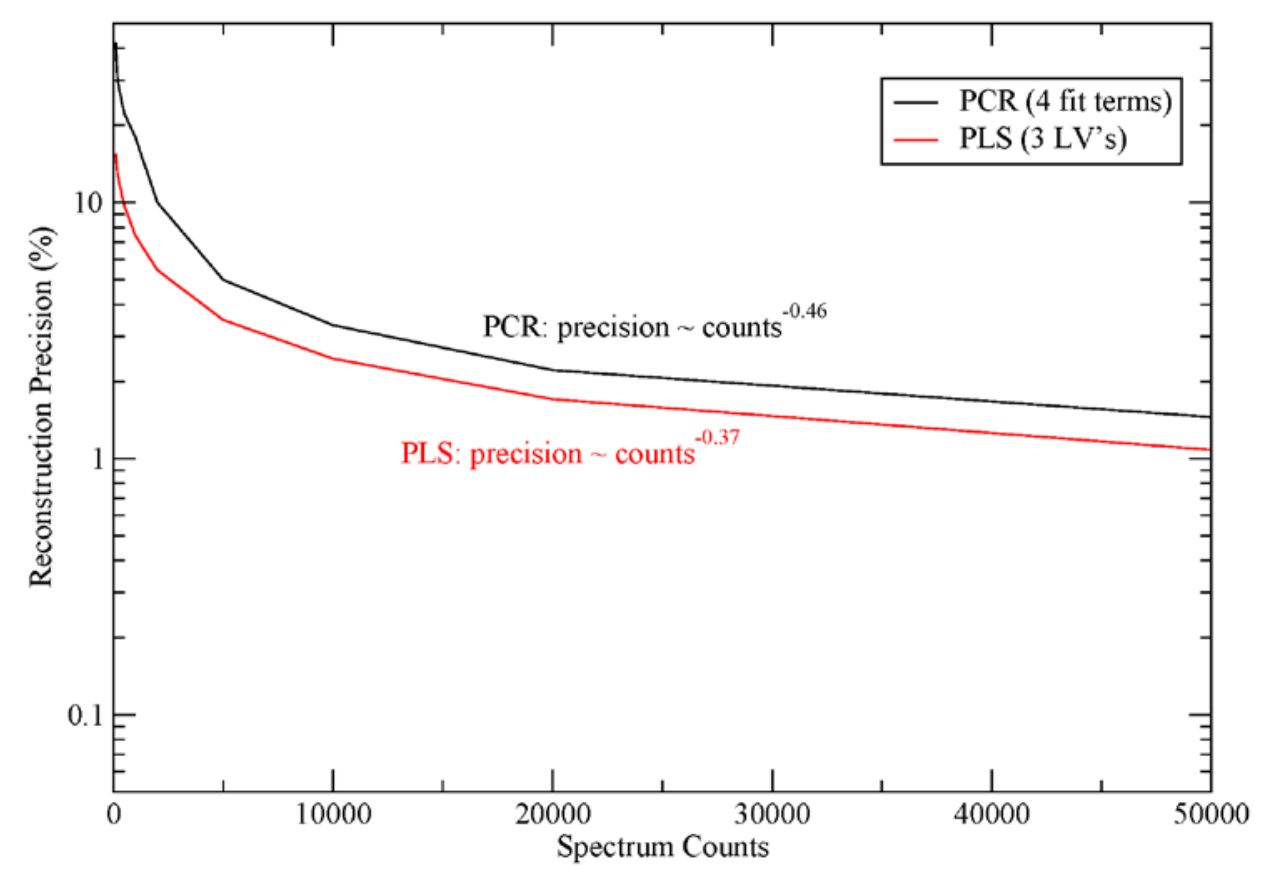

Figure 2-2. Statistical Monte Carlo modeling results for application of two multivariate analysis techniques to a set of simulated gamma-ray spectra. The reconstruction precision refers to the RMS fractional deviation of reconstructed from actual source-component activities for a set of 13 synthetic sources consisting of various mixtures of ${ }^{241} \mathrm{Am},{ }^{57} \mathrm{Co}$, and ${ }^{152} \mathrm{Eu}$. The curves join discrete points at integrated spectrum counts of $[100,200,500, \ldots, 10,000,20,000$, and 50,000] and are intended to guide the eye. The results of power-law fits to the sets of [counts, reconstruction precision] results are displayed on the plot. Note that an exponent of -0.5 in a power-law fit corresponds to $1 / \sqrt[V]{ }$ counts variation, as familiar from Poisson counting statistics.

\section{$\underline{\text { Impact of Nonlinear Mapping from Process Variables to Spectrum Components }}$}

Initial studies of the impact of gamma-ray counting statistics on multivariate spectrum analysis methods focused on reconstruction of underlying process variables that are related linearly to the associated radioisotopes contributing to the gamma-ray spectrum. In practice, this involves assuming that the activities, or some other appropriate weighting factor, directly proportional to an isotope's gamma-ray spectrum intensity is known for each member of the training, or calibration, sample set. In general applications of the MIP monitor, however, metrics quantifying the relevant process conditions may be nonlinearly related to the relative intensities of spectrum components. The dependence of gamma-ray intensity on spent fuel burnup (BU), for example, may follow a power-law form with exponent greater than unity for certain radionuclides. The dependence of radioisotope activity on cooling time follows an exponential decay law. FY13 studies of the impact of gamma-ray statistics on precision of processvariable reconstruction via Principal Component Regression (PCR) emphasized application of PCR when a nonlinear relationship exists between process variable and radioisotope component activity. The relevance of these studies to the MIP monitor is an enhanced understanding of the implications and limitations of the application of linear multivariate methods to process conditions in which the process variable basis (i.e. the set of variable characterizing the sample set) has a nonlinear relationship to the observed variables (i.e. the counts in a set of gamma-ray spectrum channels). 
This is a complicated problem, and the ultimate goal for the MIP project is to quantify the impact of nonlinearities on anomaly detection under process-monitor conditions. We have addressed a much simpler case in FY13 work to date. The particular case of a power law relationship between activity and $\mathrm{BU}$ was analyzed for a simplified, single-component spectrum in which the goal is to reconstruct assaysample BU from PCR as calibrated using a set of training samples of known, specified BU. Monte Carlo modeling of this test case was repeated at values of the exponent in the BU power law varying from 1 (i.e. linear dependence of activity on BU) to 2 (i.e. quadratic dependence). The training-sample set consisted of notional gamma-ray spectra scaled by a radioisotope activity factor that has a power-law dependence on the BU. PCR analysis yielded a prediction of test-sample BU for independent spectra generated at BU values intermediate between elements of the calibration-sample set. In each case, the mean reconstructed assay-sample BU was determined from a Monte Carlo ensemble of synthetic data sets, and the dependence of the bias in reconstructed BU was assessed as a function of the BU powerlaw exponent. The results suggest an absolute bias in the reconstructed $B U$ that varies approximately quadratically with the excess in power-law exponent above 1. Figure 2-3 displays the deviation from nominal test-sample BU (a value of 75 in arbitrary units for this example) as a function of the power-law exponent. The onset of this bias, which amounts to several percent in the reconstructed $\mathrm{BU}$ as the power-law approaches a quadratic functional form, is a consequence of the use of an inherently linear regression technique (PCR) in describing a data set with a nonlinear relationship between process variable and spectrum intensity. Ongoing FY13 work will generalize this method of quantifying PCR reconstruction bias to study the properties of reconstruction as a function of gamma-ray spectrum complexity, number of spectral components, and alternative process variable radioisotope activity relationships (e.g. cooling time and initial fuel enrichment). 


\section{Simulation of BU Reconstruction via PCR}

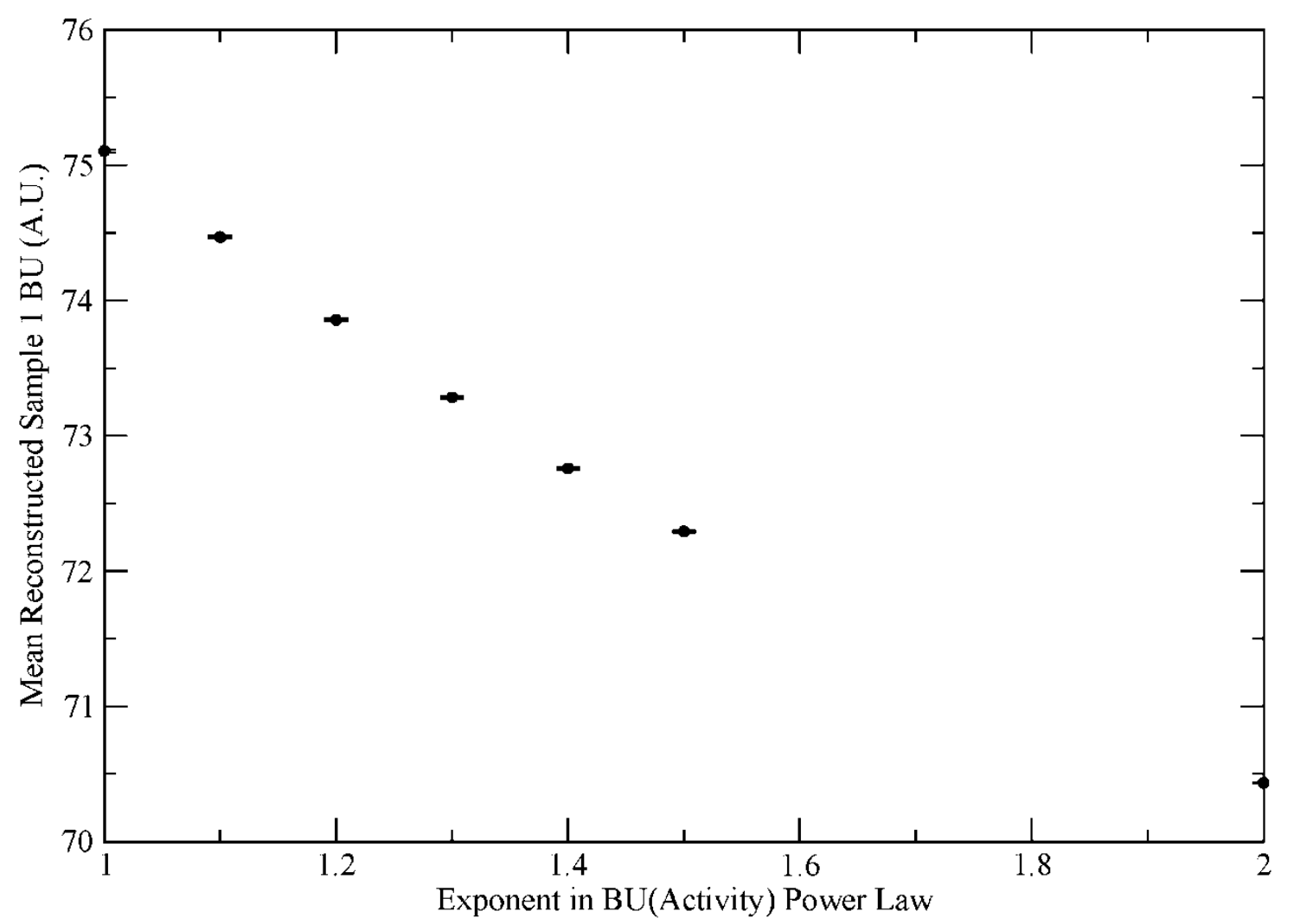

Figure 2-3. Modeled dependence of reconstructed sample BU bias on the nonlinearity in the underlying law mapping BU to spectrum component intensity.

\subsection{Task 2: Evaluating the Efficacy and Applicability of the MIP Monitor to Pyroprocessing}

A major FY13 effort is to evaluate the applicability of the MIP Monitor approach to pyroprocessing streams. This assessment began with literature survey to understand the pyroprocessing process and flowsheet. Based on this initial assessment, the MIP Monitor should be applicable to key locations in the pyroprocessing process stream. To confirm this, two types of evaluation were performed.

The first assessment evaluates the ability to detect anomalous behavior. Gamma spectra for nominal conditions at key locations in the pyroprocessing process were simulated to develop a multivariate model of the process. Additional normal and off-normal spectra were simulated to test the ability to differentiate nominal and anomalous operation. For this analysis, we used results from the pyroprocessing simulator, SSPM EChem, developed in FY12 at Sandia National Laboratory. This simulator, coupled with the gamma generating code Synth developed at PNNL, provided the necessary data to test the MIP Monitor with gamma spectra typical of key locations in the pyroprocessing stream. 
The second assessment evaluates the deployment of gamma spectra in a pyroprocessing environment. For this analysis, radiation transport models of the relevant locations, geometries, and materials (distributed radiological materials in an inert matrix of attenuating materials) were developed to assess the detected gamma spectra. This study included measurement scenario definitions, radiation-transport and detector-response modeling, and multivariate spectroscopic analysis. The radiation transport modeling included internal, or "self," attenuation of the gamma-ray emissions as they leave the process stream (e.g. a molten salt) and traverse pipe walls or other intervening shielding before impinging on the spectrometer.

The following sections present the work completed to perform these two evaluations and the results.

\subsubsection{Anomaly Detection in Pyroprocessing}

In reviewing the available literature on pyroprocessing, the SSPM EChem model developed at Sandia National Laboratory (Cipiti et al. 2012) was identified as a potential source of isotopic information for key locations in the pyroprocessing stream. Results of this model were provided for nine simulated PWR fuels at five key locations in the pyroprocessing stream (electrorefiner, shredded fuel input, metal waste form output, $U$ product output and U/TRU product output). The SSPM EChem model simulates the mass of elements 1-99 moving through the system; these masses can be converted to isotopics through the original ORIGEN model output, because the isotopic ratios remain constant through the chemical separation plant.

The provided ORIGEN output files and SSPM EChem model outputs were used to generate the isotopic concentrations at each of the five key locations. These isotopics were then input to SYNTH, a program developed at PNNL to simulate the measured gamma spectra for a specified source. The gamma spectra were simulated for each simulated fuel sample; in each case, the gamma source was treated as a point source $25 \mathrm{~cm}$ ( 10 inches) from the detector. Air was the only absorber between the source and detector. A coaxial HPGe detector was used, with the detector and counting characteristics given in Table 2.3. 


\begin{tabular}{|c|c|c|}
\hline \multirow{3}{*}{ End Cap } & Thickness & $0.5 \mathrm{~mm}$ \\
\hline & Material & Aluminum \\
\hline & Spacing & $0.666 \mathrm{~mm}$ \\
\hline Dead Layer & Thickness & $0.666 \mathrm{~mm}$ \\
\hline \multirow{4}{*}{ Sensor } & Diameter & $6.66 \mathrm{~cm}$ \\
\hline & Length & $6.66 \mathrm{~cm}$ \\
\hline & Efficiency & $66.60 \%$ \\
\hline & Resolution@1332 keV & $1.666 \mathrm{keV}$ \\
\hline \multirow{3}{*}{ Counting Parameters } & Zero & $0 \mathrm{keV}$ \\
\hline & Gain & $1 \mathrm{keV} / \mathrm{ch}$ \\
\hline & Quad & $0 \mathrm{keV} / \mathrm{ch}^{2}$ \\
\hline \multirow{2}{*}{ ADC } & Channels & 4096 \\
\hline & Live Time & $666 \mathrm{sec}$ \\
\hline Full Scale Energy & & $4096 \mathrm{keV}$ \\
\hline
\end{tabular}

Table 2.3. HPGe Detector and Electronics Parameters for SYNTH Gamma Spectra Simulations

In addition to the spectra representing normal operation, diversion scenarios were simulated to evaluate the ability to detect these anomalies. Two diversion scenarios were considered: first, bulk material from the U/TRU product output was removed by reducing all the isotope masses by some percentage; second, plutonium from the U/TRU product was removed by reducing only the masses of $\mathrm{Pu}$ isotopes, while leaving the other masses constant. Both of these scenarios were evaluated for diversions of $0.1,0.5,1.0,2.0$, and $5.0 \%$. A PC model of the spectra representing normal operation at the U/TRU product stage was developed using a variety of preprocessing methods, including standardization, mean centering, normalization, and combinations of the three. The diversion detection ability was then tested by projecting the anomalous spectra to the PC model and evaluating the Qresiduals and Hotelling $\mathrm{T}^{2}$ statistic; these two measures quantify how well a new observation fits into the PC model. Because only nine normal spectra were available, the PC models were limited to three principal components to avoid over-fitting. As more data becomes available for additional fuel types, the PC model may become more accurate and able to detect smaller changes. In all cases, 5\% material diversion (bulk or Pu only) is easily detected; however detection of smaller diversions is limited. Diversions of $0.1 \%$ are never detected using this model and the available data. Results of the bulk diversion are shown in Figure 2-4 for a PC model with data standardization. In this case, the $2 \%$ diversion is also easily detected by the Q-residuals, and some cases of $1 \%$ diversion can be detected. For one fuel type, even the $0.5 \%$ diversion can be detected. Applying different data preprocessing methods did not improve diversion detection. Results of the same model applied to diversion of Pu only are shown in Figure 2-5. With this model, only a few cases of $1 \%$ and $2 \%$ diversion can be detected. By first normalizing the spectra and then standardizing, the diversion detection is somewhat improved, with more cases of $2 \%$ diversion detected (Figure 2-6). The normalization in this case will remove any differences in the total number of counts by scaling the spectrum to the total counts (giving an area of 1). This may be more helpful in cases of single element diversions by removing the small, uninteresting differences in counts that would likely be due to the stochastic nature of radiation emission. In the case of bulk material diversion, these changes in total counts are precisely what we want to capture, so normalization is not helpful. 


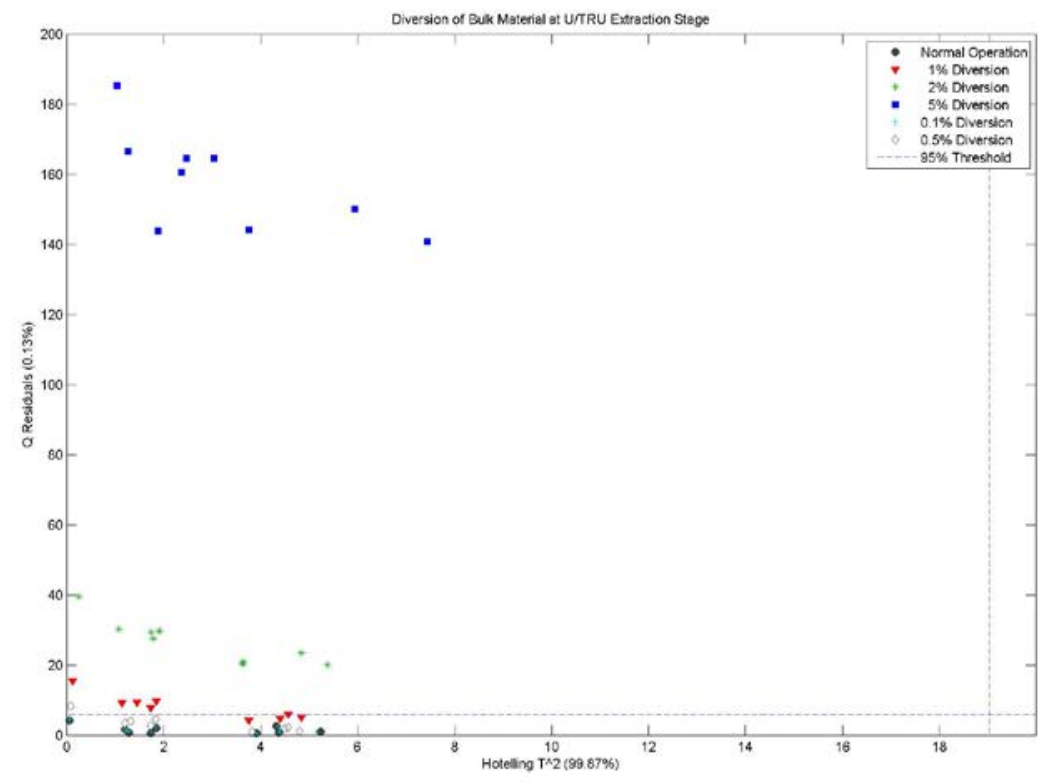

Figure 2-4. Q-residuals and $\mathrm{T}^{2}$-statistic for detection of bulk material diversion at the U/TRU product output. This model includes 3 PCs and spectrum standardization.

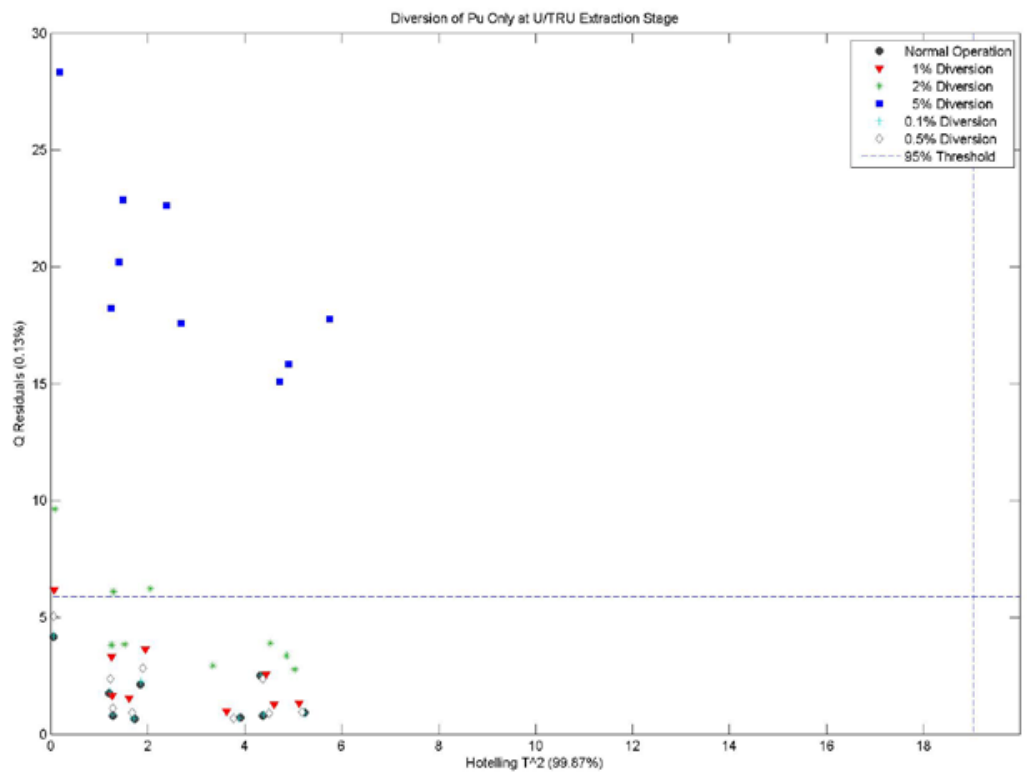

Figure 2-5. Q-residuals and $\mathrm{T}^{2}$-statistic for detection of Pu diversion at the U/TRU product output. This model includes 3 PCs and spectrum standardization. 


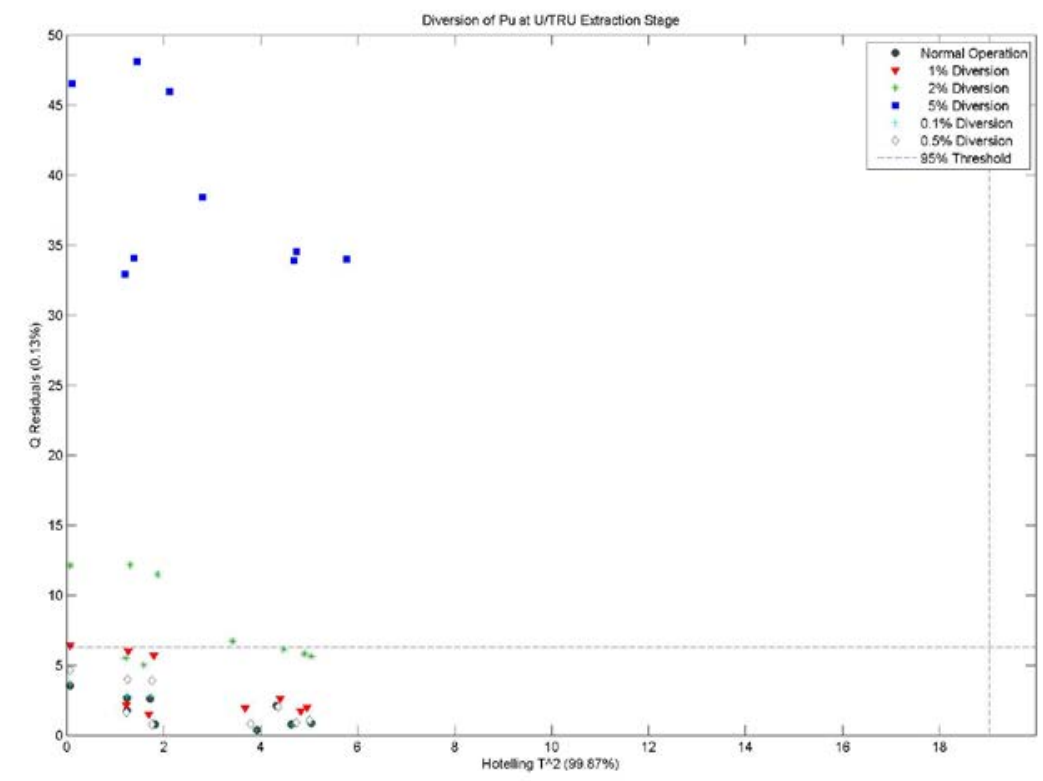

Figure 2-6. Q-residuals and $\mathrm{T}^{2}$-statistic for detection of Pu diversion at the U/TRU product output. This model includes 3 PCs and spectrum normalization followed by standardization.

\subsubsection{Simulation of Pyroprocessing Process Monitoring with a Medium-resolution Spectrometer}

The anomaly detection modeling described in Section 2.2.1 above addressed the use of a highresolution (HPGe) spectrometer viewing a point source comprising U/TRU radioisotope inventories at a specific point in the pyroprocessing flowsheet. The simulations described in the present section complement the point-response studies in a study of extended spatial source terms (e.g. electrorefining vessels) viewed by a medium-resolution (Nal) spectrometer. The objectives of the modeling study are to (a) estimate detected signal rates in the gamma-ray spectrometer, and (b) assess the potential for multivariate techniques to indicate significant, or anomalous, changes in the radioisotope inventory.

The key qualitative features of the pyroprocessing flowsheet (in contrast to aqueous reprocessing techniques) include relatively high-activity radioisotope inventories entrained in molten salts, with typical container vessel dimensions of order $10 \mathrm{~cm}$ to 1 meter, or electrodeposited as metals on electrodes. The main challenge of assessing utility of the MIP approach as applied to pyroprocessing is to select an appropriate notional physical measurement scenario and a model of typical used-fuel radioisotope inventories present at various stages of the flowsheet. PNNL used synthetic data produced by the SSPM EChem pyroprocessing flowsheet model (developed at Sandia National Laboratory) to construct radioisotope inventories from a simulated PWR used-fuel assembly, evaluated at nine different combinations of initial enrichment and burnup. PNNL applied radiation transport modeling of a notional electrorefining vessel containing fission-product radioisotopes entrained in molten salt to 
generate simulated gamma-ray spectra in a medium-resolution $\mathrm{Nal}$ spectrometer viewing the vessel at 1-meter standoff.

PCA analysis of the resulting simulated spectra for the nine used-fuel cases indicated that differences in initial enrichment ( $1 \mathrm{wt} \%$ variation, from $2.6 \mathrm{wt} \%{ }^{235} \mathrm{U}$ to $5.4 \mathrm{wt} \%{ }^{235} \mathrm{U}$ ) and burnup $(33,45$, and $60 \mathrm{GWd}$ ) are readily distinguished from one another if random fluctuations in the used-fuel radioisotope inventories (resulting from, e.g., fuel fabrication tolerances and small systematic variations in reactor neutron flux or irradiation history) are typically of order $0.1 \%$ or less. In this case, the MIP approach could conceivably detect anomalous reactor irradiation history of a particular fuel assembly type consumed in the reprocessing flowsheet. At random radioisotope inventory variations on the order of $1 \%$ or larger, however, the multivariate analysis approach applied in this study would have trouble identifying an anomalous reactor irradiation history. The potential for distinguishing radioisotope inventories from different types of fuel assemblies, and/or different reactor types, was not evaluated in FY-2013 work and remains a topic for further investigation. The remainder of this section furnishes details supporting these conclusions.

Following a literature search aimed at broadly characterizing the pyroprocessing flowsheet (Li et al. 2005, Durkee 2012, Williamson and Willet 2011, Laidler et al. 1997, Davies and Li 2007, Benedict et al. 2007), PNNL used MCNP5 to construct a simplified electrorefining vessel model for purposes of gammaray radiation transport simulation. The vessel is a 1-m diameter by 1-m height cylinder with Fe walls of thickness $1 / 8^{\prime \prime}(3.2 \mathrm{~mm})$ containing $\mathrm{KCl}$ molten salt at a density of $1.7 \mathrm{~g} / \mathrm{cm}^{3}$; see Figure $2-7 . \mathrm{A} 3^{\prime \prime} \times 3^{\prime \prime}$ $(7.62 \mathrm{~cm} \times 7.62 \mathrm{~cm})$ cylindrical Nal gamma-ray sensor, clad in 1-mm of Al, views the vessel at a standoff distance of 1-m from the outer wall of the vessel. The gamma-ray emission source term was derived from the SSPM EChem model (Cipiti et al. 2012) for all radioisotopes present in EChem' s electrorefiner vessel inventory (including U/TRU and fission products) using the SuperSYNTH program (Hensley **) to create the input gamma-ray distribution for MCNP5. All gamma-ray emission lines contributing $99.99 \%$ of the total emission intensity were retained in the source term used for the MCNP5 simulation. 


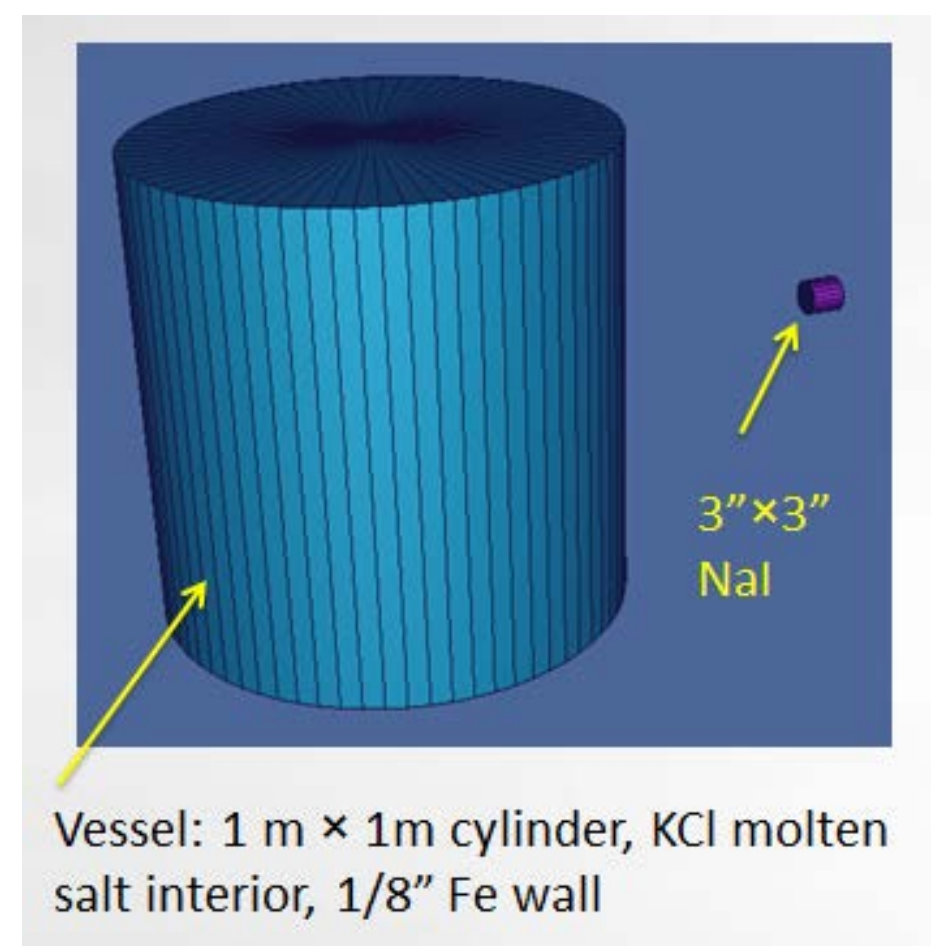

Figure 2-7. Schematic of MCNP5 electrorefining vessel model.

Figure 2-8 displays typical simulated Nal spectra produced by the MCNP5 model. The modeled spectrum is normalized to the total electrorefiner radioisotope inventory activity. The typical response rates are of order $10^{9}$ counts per second per $3-\mathrm{keV}$ spectrum bin. Note that this count rate, registered by an uncollimated Nal detector in the MCNP5 model, is much too high for a realistic detector working in pulse-processing mode. In an actual deployment, the spectrometer would view the electrorefining vessel through pinhole collimation (e.g. a narrow channel through a thick block of high-Z material) in order to reduce the data collection rate to tolerable levels (several 10's to several 100's of kHz). PNNL did not pursue collimation design given the emphasis on multivariate analysis techniques in the MIP project. For the purposes of the multivariate spectrum analysis, we assume that high count-rate mitigation strategies have been implemented and that the spectra recorded have statistical uncertainties (i.e. Poisson counting variation) small compared to the systematic differences in spectrum shape induced by differing radioisotope inventories. Figure 2-9 illustrates in greater detail these typical spectrum shape differences for different used-fuel conditions in the EChem flowsheet model. The plots correspond to the bin-wise ratio of the two higher-burnup spectra to the lowest-burnup spectrum at initial fuel enrichment of $4 \%$. As indicated in the figure by the dotted lines, the mean spectrum intensity roughly follows the total radioisotope activity inventories present in the EChem model. In addition to this mean intensity variation, differences in fuel burnup induce small (of order 1\%) energy-dependent shape distortions in the medium-resolution spectrum. Because PCA analysis is applied to the absolute (rather than unit-normalized) spectra in the present study, both the mean intensity variation and the shape differences in the spectrum are the key physical signature exploited in the multivariate analysis. 
Electrorefining Model: Simulated NaI Sensor Response

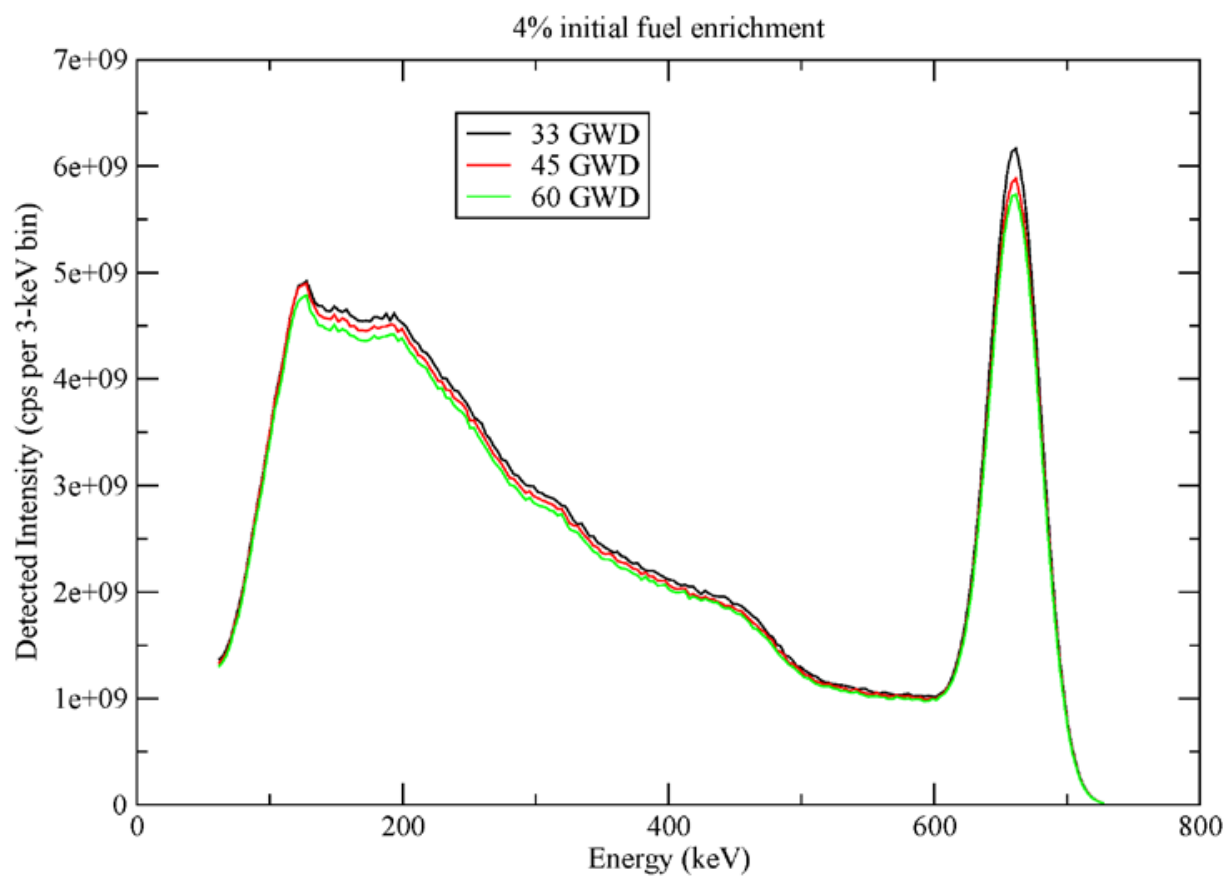

Figure 2-8. Simulated Nal gamma-ray spectrometer responses to the electrorefiner pyroprocessing inventories predicted by the SSPM EChem model at an initial fuel enrichment of $4 \%$ and burnup of 33 , 45 , and $60 \mathrm{GWd}$.

Bin-wise Spectrum Ratios, Simulated NaI Response

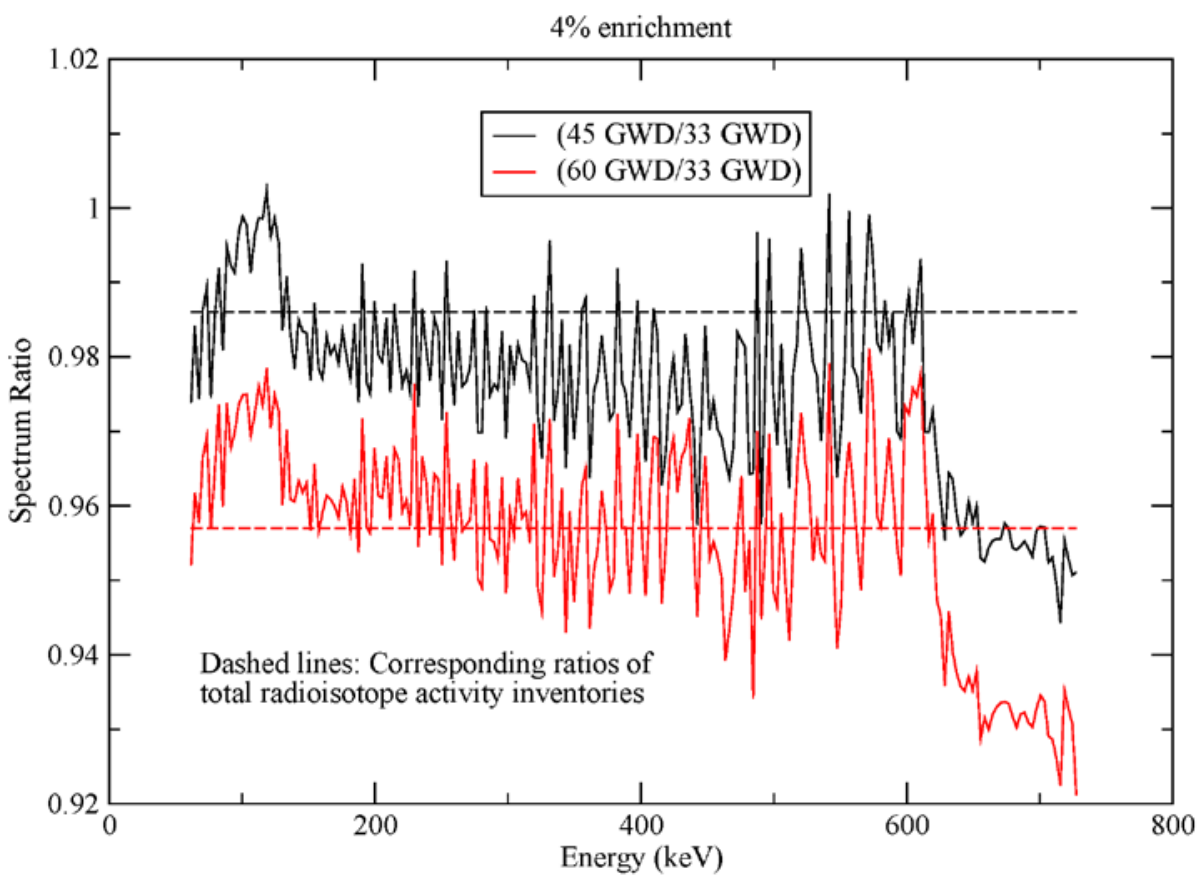

Figure 2-9. Simulated Nal energy spectrum shape differences as a function of fuel burnup. Deviations of this spectrum from a constant value indicate energy-dependent variation in the spectrum shape as the used fuel burnup varies. 
Multivariate analysis proceeded as follows. For each of nine used-fuel conditions (with initial enrichment and burnup varied), an ensemble of 100 synthetic spectra was generated by randomly and independently varying the electrorefiner radioisotope inventories about the mean, or central, values in the corresponding EChem model. Each radioisotope in a given model was assumed to be normally (i.e. Gaussian) distributed, corresponding to small random variations in fuel fabrication tolerances, small variations in neutron flux distributions and irradiation history in the reactor (for given nominal initial enrichment and burnup), and any other class of small systematic effect that would not normally constitute a process-stream anomaly. The corresponding distribution of gamma-ray intensities were then used to produce ensembles of simulated gamma-ray spectra in the Nal. PCA analysis was applied to the set of nine Nal gamma-ray spectra corresponding to the central, or nominal, radioisotope inventories. The resulting basis vectors from the PCA analysis were then used to project the set of $9 \times 100$ randomly-sampled spectra into a two-dimensional PCA score space. This procedure was repeated for relative standard deviations (RSDs) of $0.1 \%$ and $1 \%$ in the electrorefiner radioisotope inventories.

Figure 2-10 and Figure 2-11 display the results of the PCA analysis for electrorefiner radioisotope inventory RSDs of $0.1 \%$ and $1.0 \%$, respectively. Note that each cluster of black points in the scatter plots corresponds to a distinct set of 100 detected gamma-ray distributions as generated using the randomlysampled radioisotope inventories. The plot in Figure 2-10 includes a typical $90 \%$ confidence ellipse, or confidence region, for identification of spectra corresponding to a specific nominal used-fuel model. The plot shows that, at $0.1 \%$ radioisotope inventory RSD, the $90 \%$ confidence region is smaller than, or comparable to, the separation between distinct nominal used-fuel radioisotope models. The conclusion from this observation is that distinct used-fuel conditions (corresponding to intervals of initial fuel enrichment of order $1 \%$ and differences in burnup of order 12 to $15 \mathrm{GWd}$ ) should be readily discernible via PCA analysis relative to small, "uninteresting" random variations in radioisotope inventory of order $0.1 \%$ arising from fuel fabrication tolerances and variable irradiation history conditions. Figure 2-11 illustrates the contrast in predicted MIP performance when the order of magnitude of the electrorefiner radioisotope inventory variations is $1 \%$. At $1 \%$ relative standard deviation, the separation of central models in the PCA space is typically smaller than the scatter over model ensembles arising from random variations within a single used-fuel model, and the clear resolution of distinct model conditions observed in Figure 2-10 degrades into a broad cloud of model points that overlap significantly in the two-dimensional PCA score space. Under these conditions, distinguishing one set of initial enrichment and burnup values from another would be difficult, if not impossible, and thus the basis for defining "anomaly" detection would have to be broadened to include a wider class of used-fuel conditions. 


\section{Simulated NaI Sensor Response, Electrorefiner Vessel Model}

Normally-distributed radioisotope activity samples, $0.1 \%$ rel. stdDev

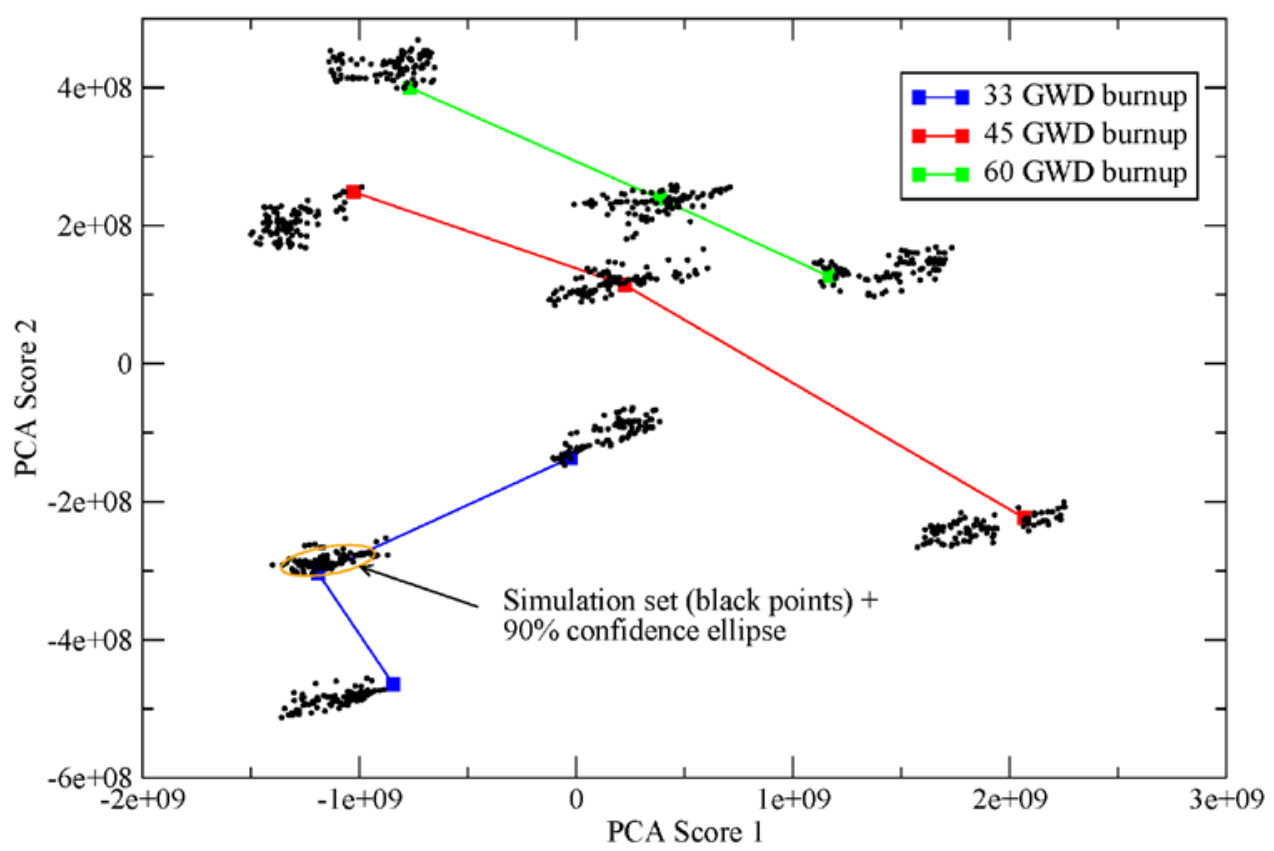

Figure 2-10. Results of PCA analysis of ensembles of simulated Nal gamma-ray spectra corresponding to randomly-sampled pyroprocessing electrorefiner radioisotope inventories of standard deviation $\mathbf{0 . 1 \%}$ relative to the central values in the EChem model. A typical $90 \%$ confidence ellipse for identification of a particular used-fuel model is displayed. The size of this ellipse is small compared to the separation between clusters of results for different fuel models (parameterized by initial enrichment, burnup), indicating that these different used-fuel conditions could be reliably distinguished by a mediumresolution gamma-ray spectrometer and thus could furnish the basis for detection of anomalous usedfuel composition. Curves in the plot join sets of constant burnup and are provided to guide the eye. 


\section{Simulated NaI Sensor Response, Electrorefiner Vessel Model}

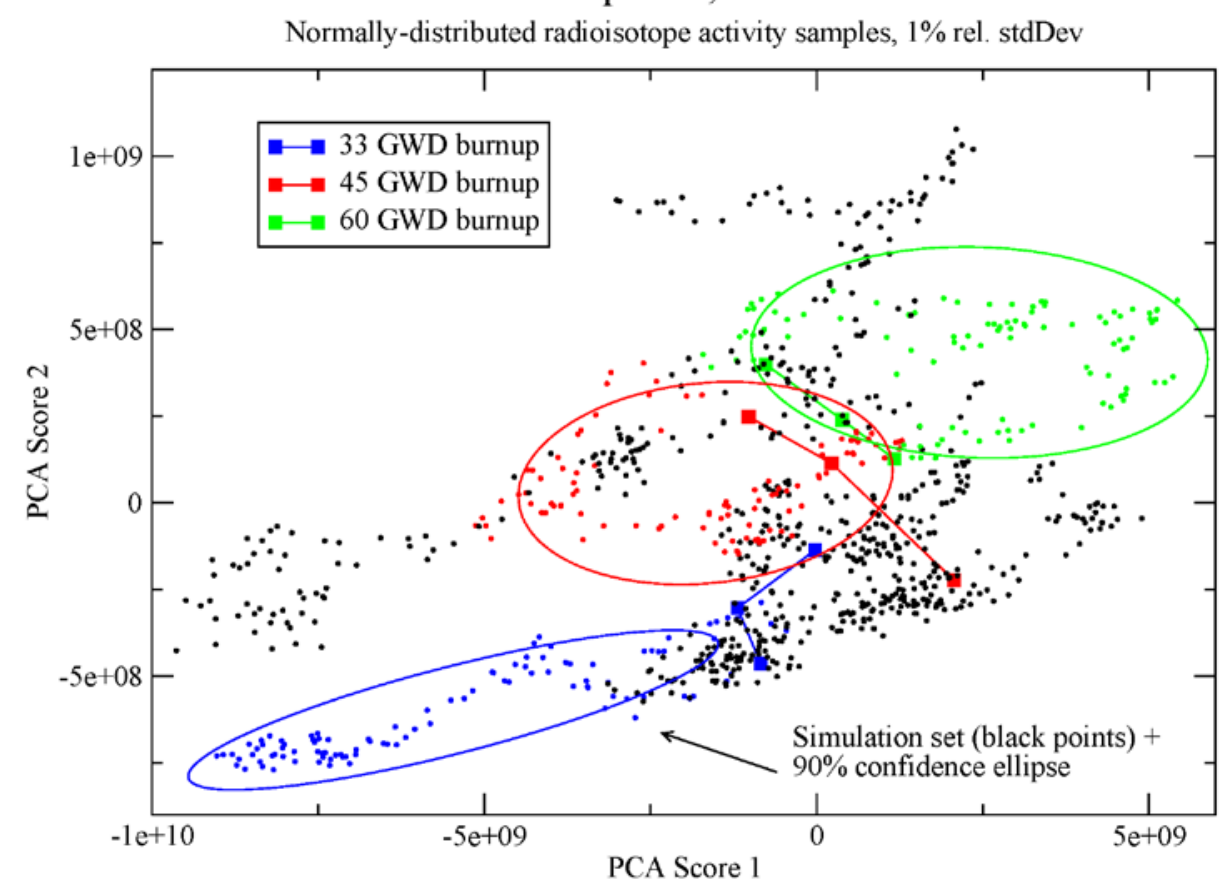

Figure 2-11. Results of PCA analysis of ensembles of simulated Nal gamma-ray spectra corresponding to randomly-sampled electrorefiner radioisotope inventories of standard deviation $\mathbf{1 \%}$ relative to the central values in the EChem model. Several confidence regions for identification of representative usedfuel models at burnups of 33,45 , and $60 \mathrm{GWd}$ are illustrated. In this case, the confidence regions are relatively large compared to the separation of clusters of points corresponding to distinct fuel models, suggesting that it would be difficult to reliably distinguish distinct fuel models (i.e. distinct initial fuel enrichments and burnups) from one another over the range of fuel enrichment and burnup considered in the study. Curves in the plot join sets of constant burnup and are provided to guide the eye.

In summary, the radiation transport modeling and PCA analysis study for pyroprocessing suggests the following results:

- Detected count rates in an uncollimated, standard-sized (e.g. 3"×3") medium-resolution gammaray spectrometer will be intolerably large when viewing a typical vessel or component in the pyroprocessing flowsheet at standoff distances of order 1 meter. Engineering shielding and collimation techniques (e.g. pinhole collimation through a thick, high-Z absorber) will be required to assure survival of the detector in the radiation environment of, e.g., an electrorefiner.

- Both mean-intensity and energy-dependent shape differences, reflecting differences in the underlying radioisotope inventories specific to the used-fuel enrichment and burnup, are visible to the eye in the simulated gamma-ray spectra registered by a notional, medium-resolution $\mathrm{Nal}$ spectrometer viewing a simplified electrorefiner vessel (size of order $1 \mathrm{~m}$ ) containing used-fuel radioisotopes entrained in a representative molten salt.

- PCA analysis applied to the simulated Nal spectra suggests that relevant differences in used-fuel characteristics (e.g. initial enrichment and burnup) are easily detected relative to small, "uninteresting" fuel-fabrication and irradiation history random variations of order $0.1 \%$. At $1 \%$ random variation, however, process-stream anomalies involving distinct used-fuel conditions for a given fuel type and reactor type will be difficult to detect. Under these conditions, generalizing the definition of "anomaly" detection to encompass a broader class of distinct fuel 
assembly types and/or reactor types may still be feasible, but in this study, only a single PWD fuel-assembly type was considered.

\subsection{Task 3: Enhanced Gamma-Ray Spectra Collection Techniques}

During FY13, the research conducted on Compton suppression and gamma-gamma coincidence has been performed at Pennsylvania State University and University of Texas at Austin; the PNNL-based effort in this task has been minimal this fiscal year. Sarah Bender (PSU) has lead development of a GEANT model of the PSU Compton suppression system. After the model has been validated with the experimental system, it can be modified to search for a more ideal detector choice and set up for spent fuel solutions measurements. The goal of the research is to develop methods to provide enhanced gamma-ray spectra that contain information that is currently hidden under the large Compton regions from the most intense gamma-rays. Her efforts have been supported mostly by her fellowship, with the project funding a small portion of her and her advisor's interactions with the MIP Monitor project and conference participation. Also in support of this work, samples of dissolved spent fuel were transferred to PSU in early FY13 following an amendment to their license that allows them to receive the samples.

\subsection{Task 4: Training of the Next Generation of Nuclear Scientists}

Although Kenneth (Kenny) Dayman (UT-Austin) and Sarah Bender (PSU) are both working on graduate research projects that benefit the MIP Monitor project, their primary support is through their respective fellowships. Kenny completed his Master's degree in May 2012, with a thesis outlining his contributions to the development of a framework for characterizing spent fuel samples. Kenny submitted a manuscript describing his M.S. work to NIM-A, and a revision was submitted in August. Sarah plans to defend her dissertation research in Fall, 2013, with both Jon Schwantes and Chris Orton serving on her dissertation committee.

In addition to the journal article describing Kenny's M.S. work, Kenny and Sarah are jointly preparing an article comparing the multivariate methods for estimating burn-up investigated in FY11 to the more conventional gamma-ray methods for burn-up analysis. Kenny presented a summary of his MIP Monitor work at the 2012 ANS Winter Meeting.

In addition, Nuclear Energy University Program proposals were submitted with University of Tennessee, Knoxville and PSU.

\subsection{Task 5: Coordinating the MIP Monitor Project with Related Activities in Other Organizations}

The MIP Monitor continues to coordinate with NA-24 through both the NA-241 Process Monitoring working group and NA-241 Safeguards Technology. A significant effort was made to leverage the $\mathrm{H}$ Canyon measurement exploration activities in NA-241 Safeguards Technology to benefit the MIP Monitor development. Jamie Coble attended a planning meeting at Savannah River Site (SRS) to leverage the testbed capabilities at H-Canyon. Beginning in FY-14, DOE-NE will fund a three year effort 
to deploy this system at SRS's H-Canyon. PNNL will develop and design a detection system that will use the MIP Monitor to analyze processing lines at SRS's domestic reprocessing facility. This deployment will provide our project with a large set of empirical gamma spectra to further develop and evaluate the multivariate approach and gain valuable insight into the technical challenges of deployment.

Collaboration with the Chinese is still possible, but little has changed on that front. Preparations for this interaction have been made, although their planned tour of PNNL has yet to occur.

\subsection{Task 6: Bench-Scale Flow Loop Experimentation and Analysis}

A bench-scale flow loop was designed and constructed in FY11 for dynamic data collection to test the near-real-time analysis of the MIP Monitor. Spectra from dilute dissolved spent nuclear fuel samples were collected using a $\mathrm{LaBr}_{3}$ detector. The flow loop design allows for the mixing of gamma signals without actually mixing the spent fuel solutions by filling various tubes with a single fuel type. Data collected in FY11 and analyzed in FY12 revealed some problems with the flow loop setup, primarily related to the inability to maintain a constant volume of dilute spent fuel in front of the detector during mixing. The bench-scale flow loop was modified in Q4 of FY12 to provide more consistent control of the volume of material in front of the detector at any given time. The updated system is shown in Figure 2-12. In addition to updating the pump and flow path to provide additional control, event mode counting was employed, meaning that each detected gamma was recorded, instead of recording the spectrum over some set time. The flow loop experiment was rerun using this set up in late FY12.

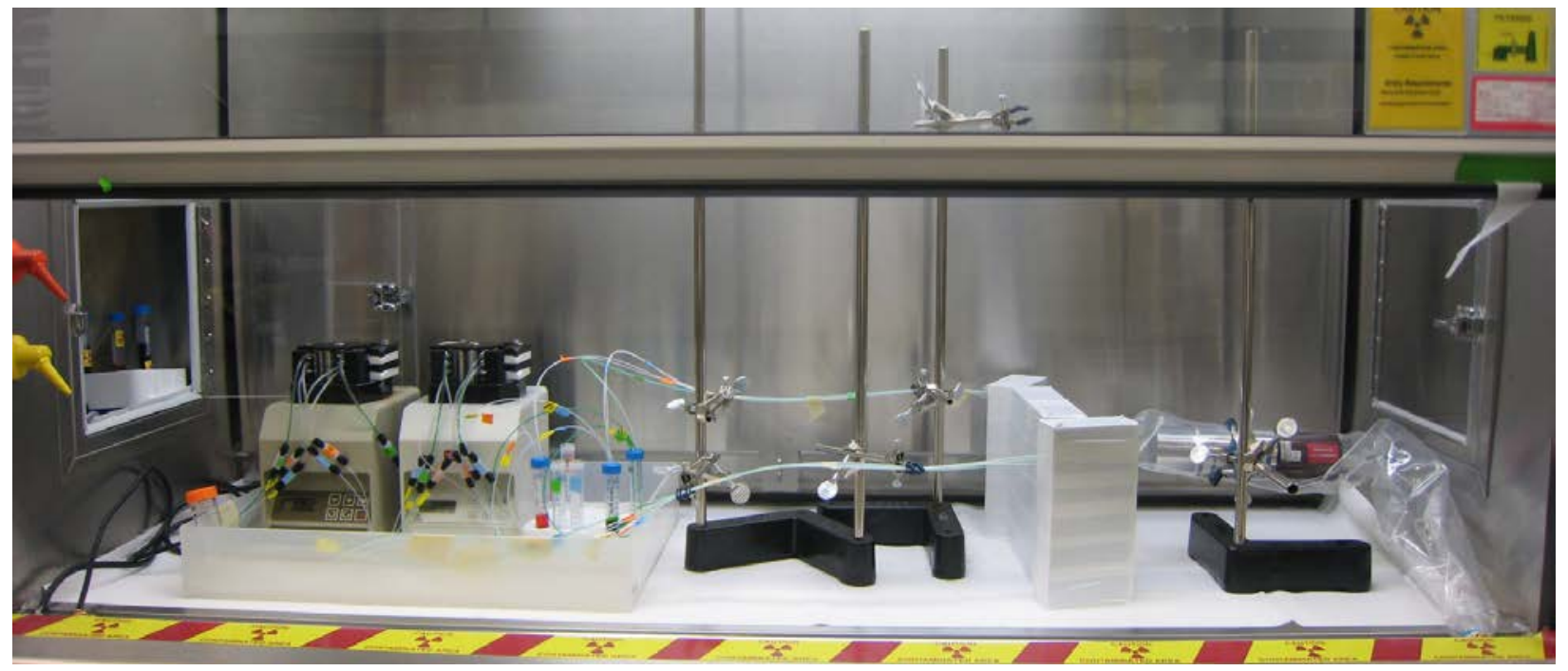

Figure 2-12. Photograph of the Updated Bench-Scale Flow Loop System

To perform dynamic fuel sample classification, the collected event-mode data were separated into 30-second spectra. Sixty- and 90-second spectra were also investigated, but no significant difference in the classification results was observed. The following results are based on the 30 -second spectra. Several preprocessing methods were employed to evaluate the effect on the PC model, including standardization, mean-centering, normalization, and combinations of the three. The results shown use 
the standardization method of the spectra prior to analysis. A PCA model of the single-fuel spectra was developed with three possible classes: ATM 105a, ATM 105p, and ATM 109 (Figure 2-13). As new spectra are collected, they are transformed to the PC-space and compared to the three fuel clusters to classify the fuel types. Figure 2-14 shows two examples of this classification. In Figure 2-14a, a progression from ATM 105p to ATM 109 is easily classified as mixtures of the two fuels. However, Figure 2-14b highlights the problem with this PC model; a progression from ATM 105a to ATM 109 travels through the cluster representing ATM 105p, which would cause the fuel to be incorrectly classified as mixtures with that fuel type. For the data evaluated, a second source of information may be necessary to accurately classify the fuel type during fuel mixing.

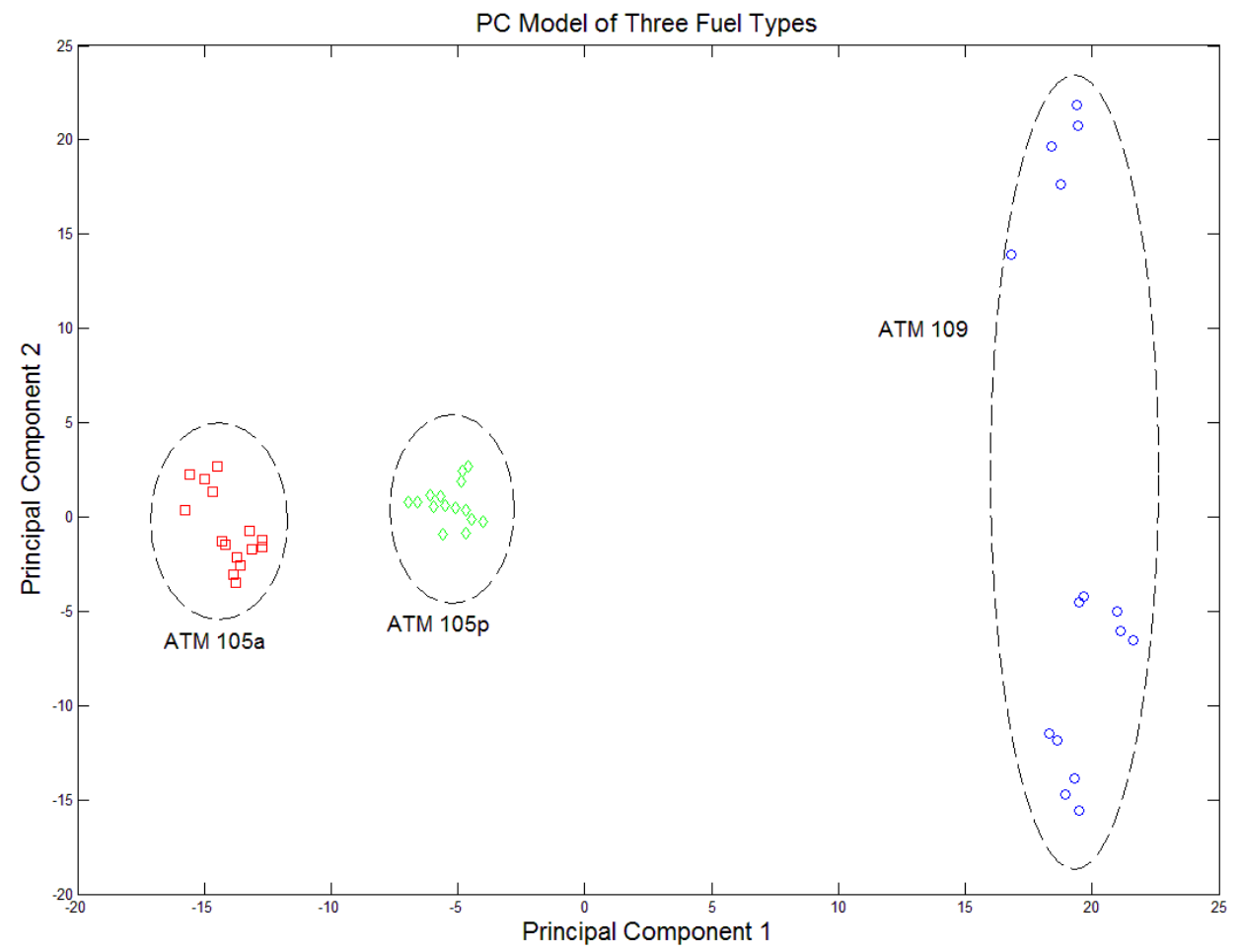

Figure 2-13. Clustering of three fuel classes in PC-space. 

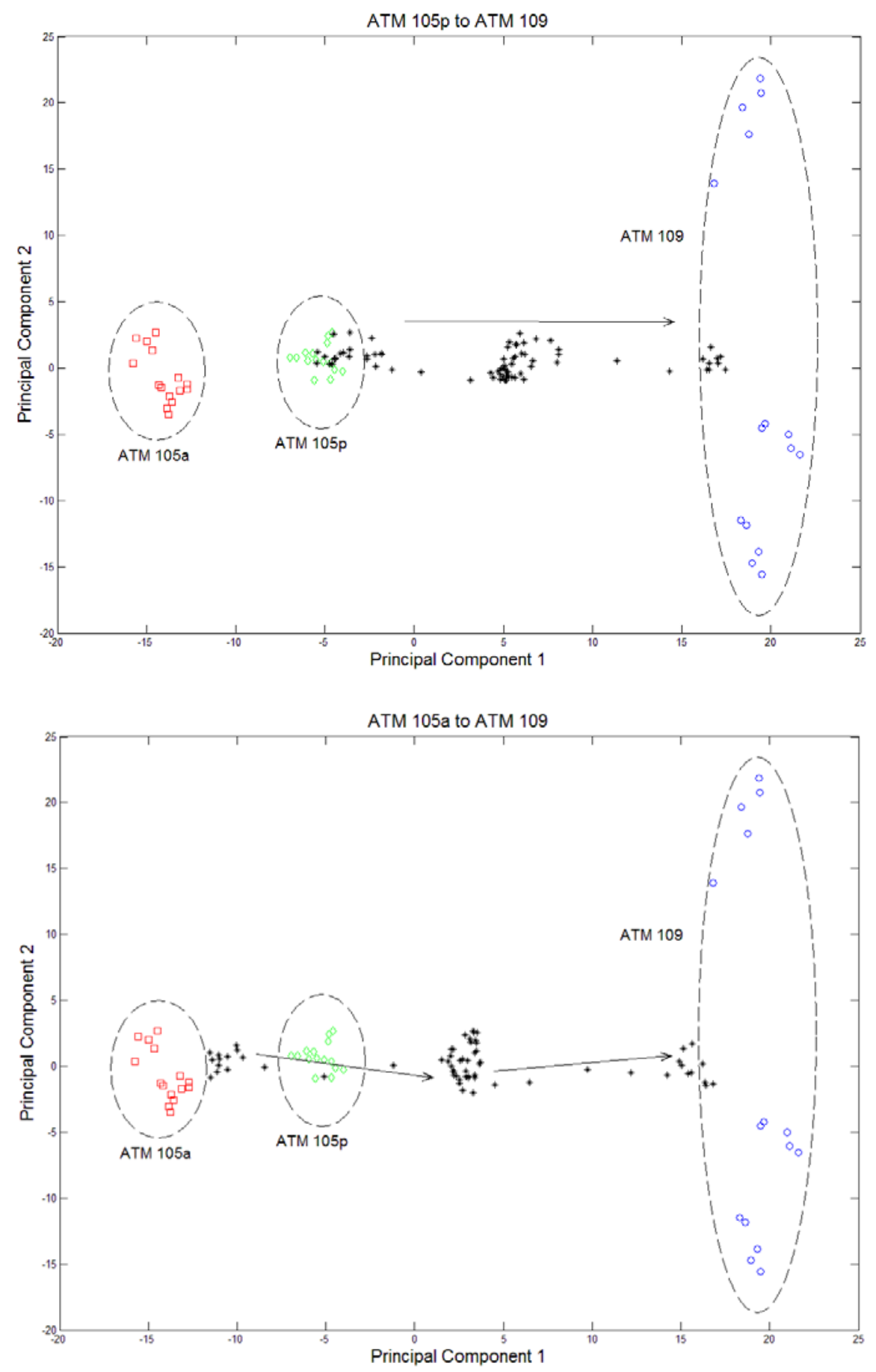

Figure 2-14. Dynamic data classification results. Fuel mixing from ATM 105p to ATM 109 can be accurately classified throughout mixing (top). Fuel mixing from ATM 105a to ATM 109 may be incorrectly classified as ATM 105p (bottom). 



\subsection{Conclusion}

The MIP Monitor project had several accomplishments during FY13, including:

- An automated algorithm for characterizing dissolved spent fuel samples according to reactor type, initial enrichment, burn up, and cooling time was developed and documented. Milestone report submitted in July 2012.

- A framework for characterizing the propagation of uncertainty in multivariate analysis of gamma spectra was developed. Experimental work to provide data to further develop and confirm the approach was performed; analysis of this data was completed in FY13.

- Spent fuel solutions were maintained to provide continued access to spent fuel solutions for testing of the MIP Monitor. Samples were transferred to PSU to support their work related to Compton suppression systems for the MIP Monitor.

- Data collected on the modified bench-scale flow loop were analyzed in early FY13. The use of event mode counting was evaluated.

- Efforts continue on several fronts to engage international partners with the intent to collaborate in order to gain access to large amounts of reprocessing data for further MIP Monitor development. To that end, Jamie Coble attended a planning meeting at Savannah River Site to discuss opportunities for deploying the MIP Monitor as part of a larger process monitoring test-bed at $\mathrm{H}$ Canyon. Project team will meet with SRNL staff in early FY-14 to develop design plans and procedures for the installation of a detector system at the H-Canyon site at SRS.

- A deployment plan was developed for DOE-NE to instrument the H-Canyon reprocessing facility at SRNL with process monitors. The MIP Monitor was included in this plan as a potential process monitoring capability.

- The MIP Monitor research and development was presented and published in several forums. Additional publications are currently being drafted or are under review. 



\subsection{Future Work}

In FY-14, PNNL will begin research and development work testing a field deployable monitoring system at SRS's H-Canyon. A detection system will be modeled and designed to acquire reprocessing spectra along at least one collection point in the reprocessing facility. PNNL will collaborate with both SRNL and University partners for this effort and the PNNL team will continue to support the training of students and young staff. Efforts will also continue to be made to secure domestic and international partners to gain access to spectral process data that can aid in the development of the MIP Monitor techniques. Significant results from all aspects from the MIP Monitor research will continue to be published in peer-reviewed journals and presented at conferences. 



\subsection{References}

Benedict M, TM Pigford and HW Levi. 1981. Nuclear Chemical Engineering. McGraw-Hill, New York.

Cipiti BB, FA Duran, B Key, Y Liu, I Lozano and R Ward. 2012. Modeling and design of integrated safeguards and security for an electrochemical reprocessing facility. Sandia National Laboratories.

Coble JB, CR Orton and JM Schwantes. 2012. Automated Characterization of Spent Fuel through the Multi-Isotope Process (MIP) Monitor. PNNL-21599, Pacific Northwest National Laboratory, Richland, Washington.

DOE. 2010. "Nuclear Energy Research and Development Roadmap." U.S. Department of Energy (DOE), Washington, DC. http://nuclear.gov/pdfFiles/NuclearEnergy Roadmap Final.pdf.

Knoll GF. 2000. Radiation Detection and Measurement. John Wiley \& Sons, New York.

Orton CR, CG Fraga, M Douglas, RN Christensen and JM Schwantes. 2009a. "Monitoring Spent Nuclear Fuel Reprocessing Conditions Non-Destructively and in Near-Real-Time Using The Multi-Isotope Process (MIP) Monitor." In 2nd JAPAN-IAEA Workshop on Advanced Safeguards Technology for the Future Nuclear Fuel Cycle, Tokai-mura, Japan. International Atomic Energy Agency, Vienna, Austria.

Orton CR, CE Rutherford, CG Fraga and JM Schwantes. 2011. The Multi-Isotope Process Monitor: Multivariate Analysis of Gamma Spectra. Deerfield, IL.

Orton CR, JM Schwantes, S Bryan, T Levitskaia, D Duckworth, M Douglas, OT Farmer, CG Fraga, S Lehn, M Liezers, S Peper and RN Christensen. 2008. "Advanced Safeguards Technology Demonstration at Pacific Northwest National Laboratory." In 49th INMM Annual Meeting. July 13-17, 2008, Nashville, Tennessee. Institute of Nuclear Materials Management (INMM), Deerfield, Illinois.

Orton CR, JM Schwantes, CG Fraga, M Douglas and RN Christensen. 2009b. "Experimental Validation of the Multi-Isotope Process Monitor Concept." In GLOBAL 2009, Paris, France. American Nuclear Society, La Grange Park, Illinois.

OTA. 1995. Nuclear Safeguards and the International Atomic Energy Agency. Report No. OTA-ISS-615, Office of Technology Assessment (OTA), Washington, D.C.

Schwantes JM, M Douglas, CR Orton, CG Fraga and RN Christensen. 2008. "Multi-Isotope Process (MIP) Monitor: a Near-Real-Time Monitor for Reprocessing Facilities." In Transactions from the Annual Meeting, Anaheim, California. American Nuclear Society, La Grange Park, Illinois.

Schwantes JM, CR Orton, CG Fraga, M Douglas and RN Christensen. 2009. "The Multi-Isotope Process (MIP) Monitor: A Near-Real-Time, Nondestructive, Indicator of Spent Nuclear Fuel Reprocessing Conditions." In 50th INMM Annual Meeting. July 12-16, 2009, Tucson, Arizona.

Smith LE, JM Schwantes, JJ Ressler, M Douglas, KA Anderson, CG Fraga, PC Durst, CR Orton and RN Christensen. 2007. "Next Generation On-line MC\&A Technologies for Reprocessing Plants." In GLOBAL 2007 Conference on Future Nuclear Energy System. September 9-13, 2007, Boise, Idaho. American Nuclear Society, La Grange Park, Illinois. 



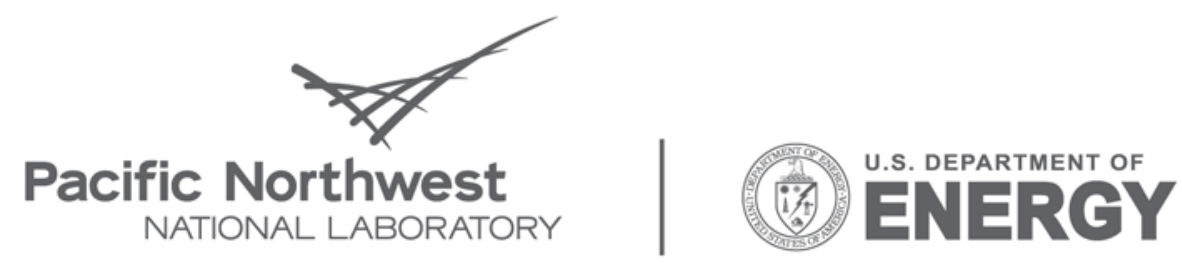

Proudly Operated by Battelle Since 1965

902 Battelle Boulevard

P.O. Box 999

Richland, WA 99352

1-888-375-PNNL (7665)

www.pnl.gov 